\title{
Nucleophilic Acyl Substitutions of Anhydrides with Protic Nucleophiles Catalyzed by Amphoteric, Oxomolybdenum Species
}

\author{
Chien-Tien Chen,* Jen-Huang Kuo, Vijay D. Pawar, Yogesh S. Munot, Shiue-Shien Weng, \\ Cheng-Hsiu Ku, and Cheng-Yuan Liu \\ SUPPORTING INFORMATION
}

Representative experimental procedures, spectral data, and ${ }^{1} \mathrm{H}$ and ${ }^{13} \mathrm{C}$ NMR spectra for all the acylation products are included (148 pages).

General. ${ }^{1} \mathrm{H}$ NMR and ${ }^{13} \mathrm{C}$ NMR spectra were recorded in deuterochloroform with chloroform as an internal reference unless otherwise stated. Chemical shifts are reported in ppm $(\delta)$. Coupling constants, $J$, are reported in Hz. Infrared spectra were reported with peaks in units of $\mathrm{cm}^{-1}$ with the following relative intensities: br (broad), s (strong 67-100 \%), m (medium 33-67 \%), or w (weak 0-33\%). Mass spectra were recorded with an ionization voltage of 70 or $20 \mathrm{eV}$ unless otherwise stated. Combustion analyses were performed by the Northern Instrument Center of Taiwan. Fast atom bombardment (FAB) and electrospray (ESI) mass spectra were reported with data in the form $\mathrm{m} / \mathrm{e}$ (intensity relative to base peak). Gel permeation chromatography (GPC) data were obtained by using THF as eluting solvent at room temperature and a polystyrene calibration curve for analyses. Analytical TLC was visualized with UV light or with phosphomolybdic acid (PMA) and $\mathrm{KMnO}_{4}$ staining agents. Column (flash) chromatography was performed using 32-63 $\mu \mathrm{m}$ silica gel. Solvents for extraction and chromatography were reagent grade. Dichloromethane was dried over $\mathrm{CaH}_{2}$ before use. THF was dried over Na with benzophenone-ketyl intermediate as indicator. All reactions were run under nitrogen and the acylation products were isolated as chromatographically pure materials. The $\mathrm{VOX}_{2}$ series of compounds (brand name as Clip-all ${ }^{\circledR}$ series, US patent \# 6,541,659 B1, 2003) is now available directly from the institution (e-mail: chefv043@ scc.ntnu.edu.tw). 


\section{General procedure for acylation reactions}

In a dry 50-mL, two-necked, round-bottomed flask was placed $\mathrm{MoO}_{2} \mathrm{Cl}_{2}(1.9 \mathrm{mg}, 0.01 \mathrm{mmol})$ in $3 \mathrm{~mL}$ of anhydrous solvent $\left(\mathrm{CH}_{2} \mathrm{Cl}_{2}\right.$ was used unless it is stated otherwise). To the above solution, anhydride $(1.5 \mathrm{mmol})$ was slowly added at ambient temperature. After for $30 \mathrm{~min}$, a solution of nucleophile (1.0 mmol in $\left.\mathrm{CH}_{2} \mathrm{Cl}_{2}, 2 \mathrm{~mL}\right)$ was slowly added to the above bluish solution and the reaction mixture was stirred for indicated time periods. After completion of the reaction as monitored by TLC, the reaction mixture was quenched with cold, saturated aqueous $\mathrm{NaHCO}_{3}$ solution $(5 \mathrm{~mL})$. For the acylation of $\beta$-hydroxy ketones or esters, ice-cold water was used to quench the reaction to prevent $\beta$-elimination. The separated organic layer was washed with brine, dried $\left(\mathrm{MgSO}_{4}\right)$, filtered, and evaporated. The crude product was purified by column chromatography on silica gel if required (in most of the acetylation reactions, essentially pure material was obtained without further purification). The product obtained was characterized by routine spectroscopic methods.

\section{General procedure for catalyst recovery}

In a dry 250-mL, two-necked, round-bottomed flask was placed $\mathrm{MoO}_{2} \mathrm{Cl}_{2}(100 \mathrm{mg}, 0.5 \mathrm{mmol})$ in $50 \mathrm{~mL}$ of anhydrous solvent $\left(\mathrm{CH}_{2} \mathrm{Cl}_{2}\right.$ was used unless it is stated otherwise). To the above solution, anhydride $(75 \mathrm{mmol})$ was slowly added at ambient temperature. After for $30 \mathrm{~min}$, a solution of nucleophile (50 mmol in $\mathrm{CH}_{2} \mathrm{Cl}_{2}, 20 \mathrm{~mL}$ ) was slowly added to the above bluish solution and the reaction mixture was stirred for indicated time periods. After completion of the reaction as monitored by TLC, the reaction mixture was quenched with ice-cold water (100 $\mathrm{mL})$. The separated aqueous layer was concentrated by rotatory evaporator at $40{ }^{\circ} \mathrm{C}$. Subsequently, the recovered catalyst was dried in vacuo at ambient temperature for 2 hours to get bluish solid (100 $\mathrm{mg}$ ) in essentially quantitative recovery.

\footnotetext{
2-Phenylethyl Acetate ${ }^{1}$

Data: ${ }^{1} \mathrm{H}$ NMR $\left(200 \mathrm{MHz}, \mathrm{CDCl}_{3}\right)$ 7.34-7.22 (m, 5H), $4.30(\mathrm{t}, J=7.2,2 \mathrm{H})$, $2.95(\mathrm{t}, J=7.2,2 \mathrm{H}), 2.05\left(\mathrm{~s}, 3 \mathrm{H}, \mathrm{CH}_{3}\right) ;{ }^{13} \mathrm{C} \mathrm{NMR}\left(50 \mathrm{MHz}, \mathrm{CDCl}_{3}\right)$ 171.96, 137.80, 128.85, 128.47, 126.53, 64.89, 35.07, 20.93; TLC R $\mathrm{R}_{f} 0.62$ (EtOAc/hexane, 1/ 20).
}

Trifluoroacetic acid phenethyl ester ${ }^{2}$ 


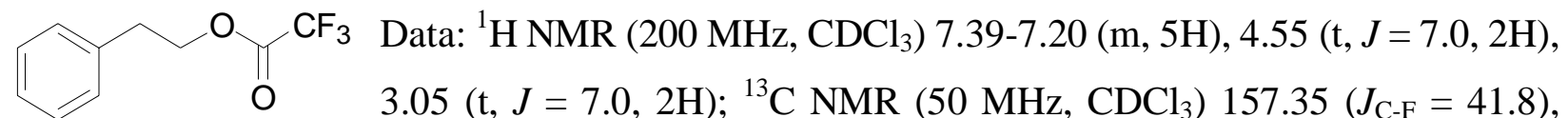
136.35, 128.87, 128.69, 127.01, $114.68\left(J_{\mathrm{C}-\mathrm{F}}=283.7\right), 68.17,34.38 ; \mathrm{MS}(70 \mathrm{eV}) 218\left(\mathrm{M}^{+}, 60\right)$, 206 (10), 192 (15), 191 (64), 178 (12), 151 (12), 141 (16), 131 (100), 124 (16); TLC R 0.31 (EtOAc/hexane, 1/20).

\section{Chloroacetic acid phenethyl ester ${ }^{3}$}

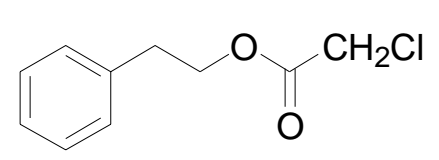

Data: ${ }^{1} \mathrm{H}$ NMR $\left(400 \mathrm{MHz}, \mathrm{CDCl}_{3}\right)$ 7.35-7.23 (m, 5H), $4.42(\mathrm{t}, J=6.8$, $2 \mathrm{H}), 4.04(\mathrm{~s}, 2 \mathrm{H}), 2.99(\mathrm{t}, J=6.8,2 \mathrm{H}) ;{ }^{13} \mathrm{C} \mathrm{NMR}\left(100 \mathrm{MHz}, \mathrm{CDCl}_{3}\right)$ 167.09, 137.10, 128.8, 128.47, 126.64, 66.42, 40.72, 34.79; MS (FAB) 265 (100), $221\left(\mathrm{M}+\mathrm{Na}^{+}, 51\right) ;$ TLC $\mathrm{R}_{f} 0.31$ (EtOAc/hexane, 1/20).

\section{Butanoic acid phenethyl ester ${ }^{4}$}

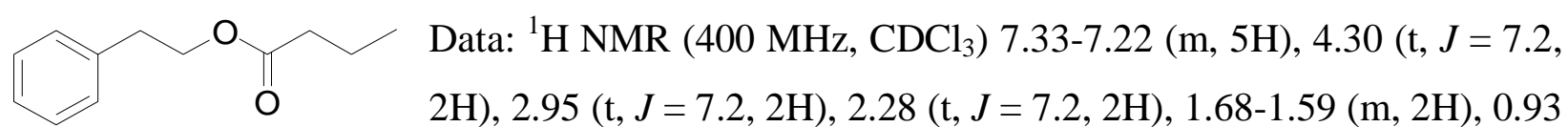
$(\mathrm{t}, J=7.6,2 \mathrm{H}) ;{ }^{13} \mathrm{C}$ NMR $\left(100 \mathrm{MHz}, \mathrm{CDCl}_{3}\right)$ 173.55, 137.85, 128.85, 128.42, 126.47, 64.62, 36.15, 35.12, 18.35, 13.59; IR $\left(\mathrm{CH}_{2} \mathrm{Cl}_{2}\right) 3061$ (s), 2988 (s), 1730 (s), 1423 (s), 1281 (s), 1181 (s), 1089 (m), 991 (m), 897(s), 764 (s), 454 (w); MS (70 eV) $192\left(\mathrm{M}^{+}, 4\right), 149$ (11), 120 (9), 104 (100), 91 (89), 77 (12), 71 (52), 57 (55); TLC R 0.32 (EtOAc/hexane, 1/20).

\section{Isobutyric acid phenethyl ester}

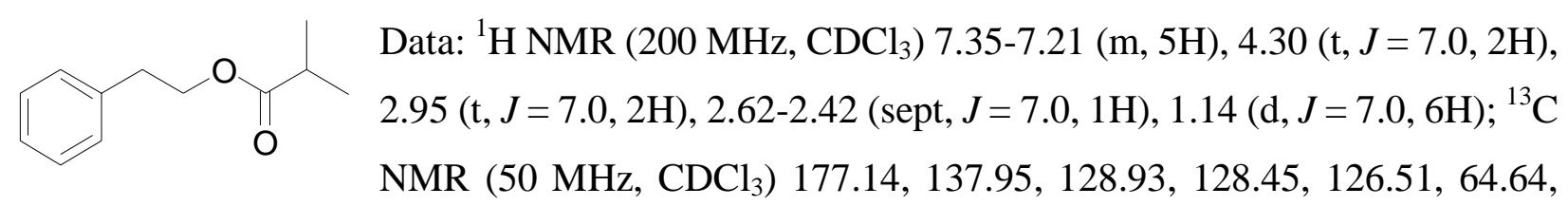
35.05, 33.90, 18.82; MS (20 eV) 193 (M+1+, 92), 191 (78), 190 (35), 188 (14), 184 (25), 183 (46), 178 (37), 175 (86), 137 (29), 168 (46); TLC R 0.36 (EtOAc/hexane, 1/20).

\section{2-Phenylethyl 2, 2-Dimethylpropanoate ${ }^{6}$}

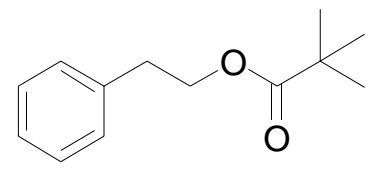

Data: ${ }^{1} \mathrm{H}$ NMR $\left(200 \mathrm{MHz}, \mathrm{CDCl}_{3}\right)$ 7.34-7.24 (m, 5H), $4.31(\mathrm{t}, J=7.0$, $2 \mathrm{H}), 2.96(\mathrm{t}, J=7.0,2 \mathrm{H}), 1.20\left(\mathrm{~s}, 9 \mathrm{H}, \mathrm{CH}_{3} \times 3\right) ;{ }^{13} \mathrm{C} \mathrm{NMR}\left(50 \mathrm{MHz}, \mathrm{CDCl}_{3}\right)$ $178.31,137.92,128.87,128.32$, 126.39, 64.72, 38.6, 35.09, 27.08; TLC R 0.54 (EtOAc/hexane, 1/20). 
Carbonic acid tert-butyl ester phenethyl ester ${ }^{7}$

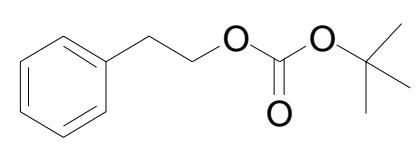

Data: ${ }^{1} \mathrm{H}$ NMR $\left(200 \mathrm{MHz}, \mathrm{CDCl}_{3}\right)$ 7.32-7.22 (m, 5H), 4.29 (t, $J=7.4$, $2 \mathrm{H}), 2.98(\mathrm{t}, J=7.4,2 \mathrm{H}), 1.49$ (s, 9H); ${ }^{13} \mathrm{C} \mathrm{NMR}\left(50 \mathrm{MHz}, \mathrm{CDCl}_{3}\right)$ 153.39, 137.32, 128.83, 128.40, 126.46, 81.67, 67.15, 35.02, 27.51; MS (70 eV) $223\left(\mathrm{M}+1^{+}, 18\right)$, 222 ( $\left.\mathrm{M}^{+}, 10\right), 207$ (14), 167 (100), 160 (10), 145 (10); TLC R 0.55 (EtOAc/hexane, 1/20).

\section{2-Phenylethyl Benzoate ${ }^{8}$}

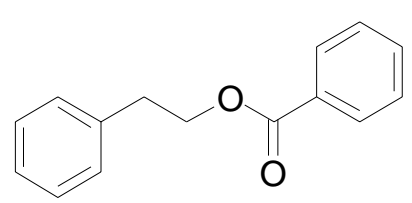

Data: ${ }^{1} \mathrm{H}$ NMR $\left(200 \mathrm{MHz}, \mathrm{CDCl}_{3}\right)$ 8.08-8.03 (m, 2H), 7.61-7.26 (m, $8 \mathrm{H}), 4.53(\mathrm{t}, J=7.0,2 \mathrm{H}), 3.11(\mathrm{t}, J=7.0,2 \mathrm{H}) ;{ }^{13} \mathrm{C} \mathrm{NMR}(50 \mathrm{MHz}$, $\left.\mathrm{CDCl}_{3}\right) 166.58,137.95,132.92,130.33,128.59,128.98,128.57,128.36$, 126.61, 65.41, 35.16; TLC R $\mathrm{R}_{f} 0.32$ (EtOAc/hexane, 1/40).

\section{Succinic acid monophenethyl ester ${ }^{9}$}

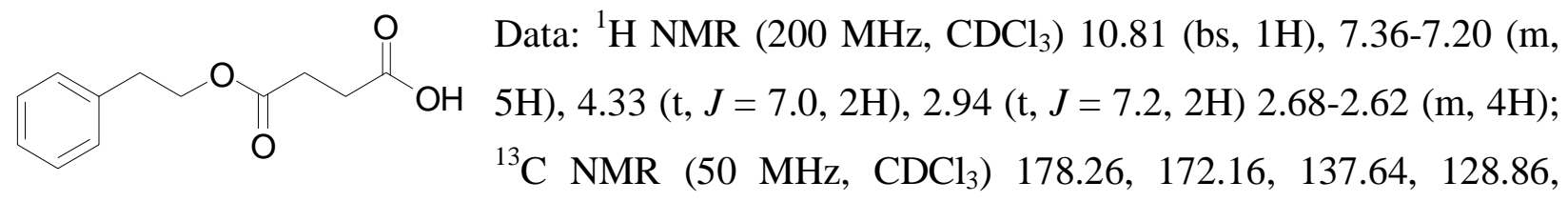
128.48, 126.55, 65.23, 34.85, 28.75, 28.69. MS (70 eV) $222\left(\mathrm{M}^{+}, 88\right), 216$ (78), 210 (78), 208 (88), 206 (56), 200 (44), 198 (100), 193 (65), 186 (44), 185 (65), 183 (88); TLC R $\mathrm{R}_{f} 0.22$ (EtOAc/hexane, $1 / 4)$.

\section{Cyclohex-4-ene-1, 2-dicarboxylic acid monophenethyl ester}

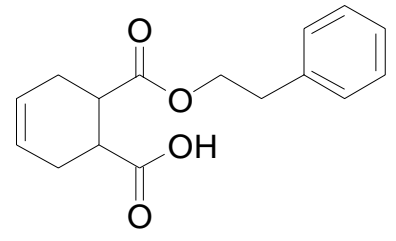

Data: ${ }^{1} \mathrm{H}$ NMR (400 MHz, $\left.\mathrm{CDCl}_{3}\right)$ 7.31-7.19 (m, 5H), 5.66 (s, 2H), 4.33-4.29 (m, 2H), 3.06-3.01 (m, 2H), $2.92(\mathrm{t}, J=6.8,2 \mathrm{H}), 2.59-2.53(\mathrm{~m}$, $2 \mathrm{H}), 2.38-2.32(\mathrm{~m}, 2 \mathrm{H}) ;{ }^{13} \mathrm{C} \mathrm{NMR}\left(100 \mathrm{MHz}, \mathrm{CDCl}_{3}\right)$ 179.42, 173.06, $137.72,128.81,128.40,126.45,125.13,65.17,39.50,39.47,34.86,25.70$,

25.50; IR ( $\left.\mathrm{CH}_{2} \mathrm{Cl}_{2}\right)$ 3495-2750 (br, m), 3055 (m), 2926 (m), 2254 (m), 1731 (s), 1710 (s), 1498 (m), 1390 (m), 1300 (m), 1270 (m), 1198 (s), 1031 (m), 911 (s), 702 (s); MS (70 eV) $275\left(\mathrm{M}+1^{+}\right.$, 6), 124 (13), 104 (100), 91 (20), 79 (48), 77 (28); TLC R 0.36 (EtOAc/hexane, 1/2). 
7-Oxa-bicyclo[2.2.1]hept-5-ene-2,3-dicarboxylic acid monophenethyl ester

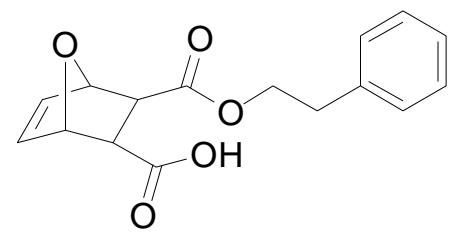

Data: ${ }^{1} \mathrm{H}$ NMR (400 MHz, d6-Acetone) 7.31-7.19 (m, 5H), 6.45 (ddd, $J=9.8,5.6,1.2,2 \mathrm{H}), 5.15(\mathrm{~s}, 1 \mathrm{H}), 5.11(\mathrm{~d}, J=1.2,1 \mathrm{H}), 4.24-4.18(\mathrm{~m}$, 2H), $2.91(\mathrm{td}, J=7.6,1.8,2 \mathrm{H}), 2.82(\mathrm{~d}, J=9.2,1 \mathrm{H}), 2.75(\mathrm{~d}, J=8.8$, $1 \mathrm{H}) ;{ }^{13} \mathrm{C}$ NMR (100 MHz, $\mathrm{d}_{6}$-Acetone) 173.04, 172.30, 139.23, 137.55, 129.84, 129.28, 127.23, 81.45, 81.08, 65.83, 47.63, 47.39, 35.53; IR $\left(\mathrm{CH}_{2} \mathrm{Cl}_{2}\right) 3498-2710$ (br, m), 3055 (s), 2685 (s), 2306 (s), 1731 (s), 1717 (s), 1424 (s), 1282 (s), 1248 (s), 1171 (s), 997 (m), 896 (s), 496 (w); MS (ESI) 333 (33), 311 (M+Na $\left.{ }^{+}, 100\right)$; TLC R 0.34 (EtOAc/hexane, 1/9).

\section{Phthalic acid monophenethyl ester and diphenethyl ester}

In a dry 50-mL, two-necked, round-bottomed flask was placed $\mathrm{MoO}_{2} \mathrm{Cl}_{2}(4 \mathrm{mg}, 0.02 \mathrm{mmol})$ in $20 \mathrm{~mL}$ of anhydrous $\mathrm{CH}_{2} \mathrm{Cl}_{2}$. To the above solution, phthalic anhydride (444.3 $\mathrm{mg}, 3.0 \mathrm{mmol}$ ) was added at ambient temperature and stirred for one hour. The reaction flask was cooled to $10{ }^{\circ} \mathrm{C}$. A solution of 2-phenylethanol $(239 \mu \mathrm{L}, 2.0 \mathrm{mmol})$ in $\mathrm{CH}_{2} \mathrm{Cl}_{2}(5 \mathrm{~mL})$ was slowly added to the above dark green solution. The resultant reaction mixture was stirred at this temperature for 78 hours. After completion of the reaction as monitored by TLC, the reaction mixture was quenched with $1 \mathrm{~N} \mathrm{HCl}(10 \mathrm{~mL})$ and stirred for one hour. The separated organic layer was washed with brine, dried $\left(\mathrm{MgSO}_{4}\right)$, filtered, and evaporated. The crude product was purified by column chromatography on silica gel (EtOAc/hexane, 1/5) to give the mono-phenethyl ester in $92 \%$ (497 $\mathrm{mg})$ along with di-phenethyl ester $(<1 \%)$.

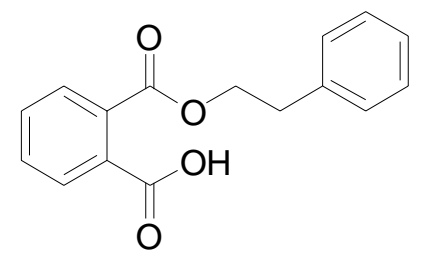

Data: ${ }^{1} \mathrm{H}$ NMR (200 MHz, $\left.\mathrm{CDCl}_{3}\right) 11.69$ (bs, 1H), 7.94-7.89 (m, 1H), 7.66-7.54 (m, 3H), 7.31-7.22 (m, 5H), $4.56(\mathrm{t}, J=7.2,2 \mathrm{H}), 3.08(\mathrm{t}, J=$ 7.2, $2 \mathrm{H}) ;{ }^{13} \mathrm{C} \mathrm{NMR}\left(50 \mathrm{MHz}, \mathrm{CDCl}_{3}\right)$ 172.04, 168.14, 137.58, 133.26, $132.06,130.82,130.18,129.75,128.92,128.69,128.49,126.55,66.24$, 34.62; IR (CCl ${ }_{4}$ 3500-2700 (br, m), 1738 (s), 1701 (s), 1600 (w), 1580 (w), 1495 (w), 1450 (w), 1409 (w), 1369 (w), 1281 (s), 1239 (m), 1125 (m), 1073 (m), 1044 (m); MS (70 eV) 271 (M+1, 100), $270\left(\mathrm{M}^{+}, 22\right), 253$ (15); TLC $\mathrm{R}_{f} 0.25$ (EtOAc/hexane,1/10).

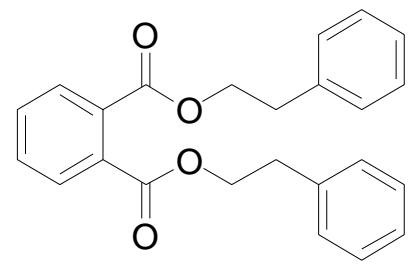

Data: ${ }^{1} \mathrm{H}$ NMR $\left(200 \mathrm{MHz}, \mathrm{CDCl}_{3}\right)$ 7.68-7.49 (m, 4H), 7.34-7.22 (m, 
$10 \mathrm{H}), 4.45(\mathrm{t}, J=7.1,2 \mathrm{H}), 3.02(\mathrm{t}, J=7.0,2 \mathrm{H}) ;{ }^{13} \mathrm{C} \mathrm{NMR}\left(50 \mathrm{MHz}, \mathrm{CDCl}_{3}\right)$ 167.55, 137.70, 132.15, 131.07, 129.00, 128.93, 128.57, 126.64, 66.05, 34.88; IR ( $\left.\mathrm{CCl}_{4}\right) 3066$ (w), $3026(\mathrm{w})$, 2923 (s), 2851 (m), 1735 (m), 1703 (w), 1600 (w), 1580 (w), 1496 (w), 1456 (w), 1408 (w), 1376 (w), 1280 (m), 1241 (m), 1121 (m), 1069 (w), 1045 (w); MS (70 eV) 375 (M+1 $\left.{ }^{+}, 100\right), 365$ (30), 361 (18), 359 (15), 351 (65), 348 (24), 345 (16), 337 (40), 325 (22); TLC R 0.57 (EtOAc/hexane, $1 / 5)$.

Phenethyloxycarbonylmethoxy-acetic acid

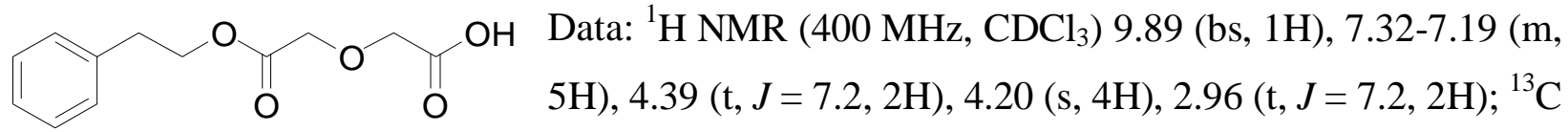
NMR (100 MHz, $\left.\mathrm{CDCl}_{3}\right)$ 173.78, 170.15, 137.11, 128.75, 128.49, 126.66, 68.18, 65.63, 34.81; IR $\left(\mathrm{CH}_{2} \mathrm{Cl}_{2}\right)$ 3500-2710 (br, m), 3056 (w), 2915 (w), 1753 (s), 1709 (s), 1606 (w), 1364 (m), 1223 (m), 1143 (s), 1053 (w), $1001(\mathrm{w})$; MS (20 eV) 239 (M+1 $\left.{ }^{+}, 6\right), 104$ (100), 91 (16); TLC R 0.36 (EtOAc/hexane, 1/4).

\section{Malonic acid monophenethyl ester}

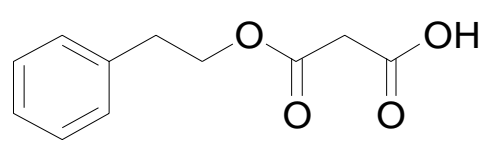

Data: ${ }^{1} \mathrm{H}$ NMR (400 MHz, $\left.\mathrm{CDCl}_{3}\right) 11.54$ (bs, 1H), 7.34-7.21 (m, $5 \mathrm{H}), 4.40(\mathrm{t}, J=6.8,2 \mathrm{H}), 3.43(\mathrm{~s}, 2 \mathrm{H}), 2.98(\mathrm{t}, J=6.8,2 \mathrm{H}) ;{ }^{13} \mathrm{C}$ NMR (100 MHz, $\left.\mathrm{CDCl}_{3}\right)$ 171.90, 166.51, 137.23, 128.85, 128.54, 126.70, 66.27, 40.90, 34.82; IR $\left(\mathrm{CH}_{2} \mathrm{Cl}_{2}\right)$ 3473-2710 (br, w), 3032 (w), 2961 (w), 1733 (s), 1605 (s), 1498 (w), 1455 (w), 1330 (m), 1154 (m), $1011(w), 878$ (w); MS (ESI) $231\left(\right.$ M $^{+}+$Na, 5), 209 $\left(\mathrm{M}+1^{+}, 11\right) ; \mathrm{TLC} \mathrm{R}_{f} 0.32$ (EtOAc/hexane, 1/4).

\section{trans-3-Phenyl-2-propen-1-yl Acetate ${ }^{10}$}

Data: ${ }^{1} \mathrm{H}$ NMR (200 MHz, $\left.\mathrm{CDCl}_{3}\right)$ 7.42-7.26 (m, 5H), 6.57 (d, $J=15.6$, $1 \mathrm{H}), 6.28(\mathrm{dt}, J=15.8,6.4,1 \mathrm{H}), 4.73(\mathrm{dd}, J=6.4,1.2,2 \mathrm{H}), 2.10(\mathrm{~s}, 3 \mathrm{H})$;

${ }^{13} \mathrm{C}$ NMR (50 MHz, $\left.\mathrm{CDCl}_{3}\right)$ 170.75, 136.20, 134.14, 128.56, 128.01, 126.55, 123.17, 64.91, 20.77; MS (70 eV) $176\left(\mathrm{M}^{+}, 44\right), 134$ (67), 133 (53), 117 (83), 115 (100), 105 (50), 92 (48), 77 (28), 57 (28); TLC R 0.29 (EtOAc/hexane, 1/20).

\section{3-Phenyl-2-propen-1-yl 2, 2-Dimethylproponate ${ }^{11}$}

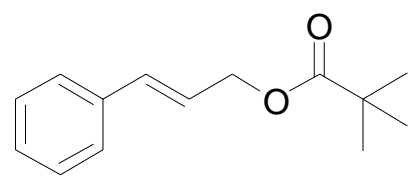

Data: ${ }^{1} \mathrm{H}$ NMR (200 MHz, $\left.\mathrm{CDCl}_{3}\right)$ 7.43-7.26 (m, 5H), 6.66 (d, $J=15.6$, 
1H), 6.36-6.22 (dt, $J=15.8,6.0,1 \mathrm{H}), 4.73(\mathrm{dd}, J=6.0,1.2,2 \mathrm{H}), 1.25(\mathrm{~s}, 9 \mathrm{H}) ;{ }^{13} \mathrm{C} \mathrm{NMR}(50 \mathrm{MHz}$, $\left.\mathrm{CDCl}_{3}\right)$ 178.34, 136.40, 133.62, 128.60, 127.98, 126.60, 123.62, 64.82, 38.72, 27.11; MS (70 eV) $218\left(\mathrm{M}^{+}, 10\right), 138$ (30), 123 (23), 117 (58), 115 (34), 96 (22), 95 (100), 85 (27), 81 (62), 67 (21), 57 (74), 55 (22); TLC R 0.35 (EtOAc/hexane, 1/20).

\section{3-Phenyl-2-propen-1-yl benzoate ${ }^{12}$}

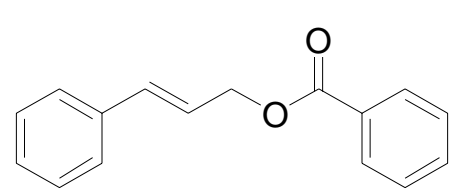

Data: ${ }^{1} \mathrm{H}$ NMR $\left(400 \mathrm{MHz}, \mathrm{CDCl}_{3}\right) 8.12(\mathrm{dd}, J=8.2,1.2,2 \mathrm{H}), 7.58(\mathrm{t}$, $J=7.4,1 \mathrm{H}), 7.48-7.26(\mathrm{~m}, 7 \mathrm{H}), 6.77(\mathrm{~d}, J=15.8,1 \mathrm{H}), 6.43(\mathrm{dt}, J=$ $15.8,6.4,1 \mathrm{H}), 5.01(\mathrm{dd}, J=6.4,1.3,2 \mathrm{H}) ;{ }^{13} \mathrm{C} \mathrm{NMR}(100 \mathrm{MHz}$ $\left.\mathrm{CDCl}_{3}\right)$ 166.25, 136.13, 134.15, 132.88, 130.12, 129.56, 128.51, 128.27, 127.98, 126.55, 123.16, 56.41; MS (20 eV) $238\left(\mathrm{M}^{+}, 15\right), 117$ (67), 105 (100), 77 (24); TLC R 0.23 (EtOAc/hexane, 1/9).

\section{Acetic acid 1-phenethyl-but-3-enyl ester ${ }^{13}$}

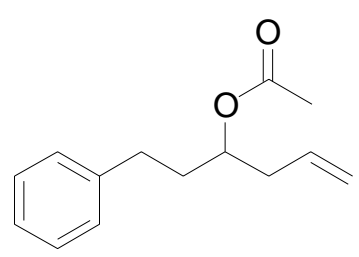

Data: ${ }^{1} \mathrm{H}$ NMR (400 MHz, $\left.\mathrm{CD}_{2} \mathrm{Cl}_{2}\right)$ 7.25-7.21 (m, $\left.2 \mathrm{H}\right), 7.15-7.12(\mathrm{~m}, 3 \mathrm{H})$, 5.76-5.69 (m, $1 \mathrm{H})$, 5.07-5.00 (m, 2H), 4.90-4.87, (m, $1 \mathrm{H}), 2.63-2.55$ (m, 2 $\mathrm{H}), 2.30-2.28,(\mathrm{~m}, 2 \mathrm{H}), 1.9$ (s, 3H), 1.85-1.79 (m, 2H); ${ }^{13} \mathrm{C} \mathrm{NMR}(100 \mathrm{MHz}$, $\left.\mathrm{CD}_{2} \mathrm{Cl}_{2}\right)$ 172.31, 143.30, 135,21, 129.86, 129.66, 127.15, 118.59, 73.26, 38.84, 35.47, 31.77, 20.90; MS (20 eV) 219 (M+1+, 30), 159 (100), 129 (12), 117 (10), HR-MS calcd for $\mathrm{C}_{14} \mathrm{H}_{18} \mathrm{O}_{2}$ : 218.1307; found: 218.1311; TLC $\mathrm{R}_{f} 0.32$ (EtOAc/hexane, 1/20).

\section{2,2-Dimethylpropanoic acid 1-phenethyl-but-3-enyl ester}

Data: ${ }^{1} \mathrm{H}$ NMR (200 MHz, $\left.\mathrm{CDCl}_{3}\right)$ 7.34-7.16 (m, $\left.5 \mathrm{H}\right)$, 5.88-5.67 (m, $\left.1 \mathrm{H}\right)$, 5.13-5.01 (m, 2 H), 2.74-2.52 (m, 2 H) 2.39-2.33 (m, 2 H), 2.00-1.84 (m, 2 $\mathrm{H}), \quad 1.24 \quad(\mathrm{~s}, \quad 9 \quad \mathrm{H}) ; \quad{ }^{13} \mathrm{C} \quad \mathrm{NMR} \quad\left(50 \quad \mathrm{MHz}, \quad \mathrm{CDCl}_{3}\right)$ $178.06,141.65,133.58,128.45,128.37,125.96,117.74,72.20,38.77$,

38.60, 35.44, 31.65, 27.13; IR ( $\left.\mathrm{CH}_{2} \mathrm{Cl}_{2}\right) 3056$ (s), 2987 (s), 1730 (m), 1550 (m), 1422 (s), 1250 (s), 1156 (m), 897 (s); MS (20 eV) 259 (M-1 $\left.{ }^{+}, 1\right), 178$ (16), 149 (76), 107 (100), 57 (12); TLC R 0.36 (EtOAc/hexane, 1/20).

\section{1-Phenethyl-but-3-enyl benzoate}

Data: ${ }^{1} \mathrm{H}$ NMR $\left(400 \mathrm{MHz}, \mathrm{CDCl}_{3}\right) 8.08(\mathrm{dd}, J=8.2,1.2,2 \mathrm{H}), 7.59(\mathrm{t}, J=$ 
7.4, 1H), 7.49-7.45 (m, 2H), 7.31-7.18 (m, 5H), 5.91-5.81 (m, 1H), 5.29-5.23 (m, 1H), 5.17-5.09 $(\mathrm{m}, 2 \mathrm{H}), 2.82-2.67(\mathrm{~m}, 2 \mathrm{H}), 2.52(\mathrm{dd}, J=7.0,6.0,2 \mathrm{H}), 2.14-2.00(\mathrm{~m}, 2 \mathrm{H}) ;{ }^{13} \mathrm{C} \mathrm{NMR}(100 \mathrm{MHz}$, $\left.\mathrm{CDCl}_{3}\right)$ 166.13, 141.46, 133.35, 132.80, 131.01, 130.55, 129.53, 128.39, 128.29, 125.90, 117.99, 73.47, 38.67, 35.36, 31.73; IR ( $\left.\mathrm{CH}_{2} \mathrm{Cl}_{2}\right) 3056$ (s), 2988 (s), 1714 (s), 1603 (m), 1424 (s), 1281 (s), 1116 (s), 1071 (m), 1026 (m), 896 (s); MS (FAB) 304 (M+Na $\left.{ }^{+}, 100\right)$; TLC R 0.28 (EtOAc/hexane, $1 / 9)$.

\section{$(2 R, 5 S)-2-I s o p r o p y l-5-m e t h y l-c y c l o h e x y l ~$ Acetate $^{14}$}

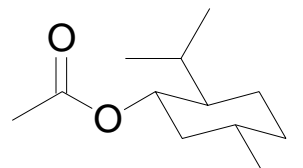

Data: ${ }^{1} \mathrm{H}$ NMR (200 MHz, $\left.\mathrm{CDCl}_{3}\right) 4.62(\mathrm{dt}, J=11.0,4.4,1 \mathrm{H}), 1.97$ (s, 3H), $1.93-1.86(\mathrm{~m}, 1 \mathrm{H}), 1.86-1.71(\mathrm{~m}, 1 \mathrm{H}), 1.70-1.54(\mathrm{~m}, 2 \mathrm{H}), 1.52-1.13(\mathrm{~m}, 2 \mathrm{H})$, $1.11-0.90(\mathrm{~m}, 2 \mathrm{H}), 0.84(\mathrm{~d}, J=7.4,6 \mathrm{H}), 0.80-0.74(\mathrm{~m}, 1 \mathrm{H}), 0.70(\mathrm{~d}, J=7.0$, 3H); ${ }^{13} \mathrm{C}$ NMR (50 MHz, $\left.\mathrm{CDCl}_{3}\right)$ 170.80, 74.13, 46.93, 34.17, 31.29, 26.22, 23.40, 21.91, 21.23, 20.64, 16.28; MS (70 eV) $198\left(\mathrm{M}^{+}, 5\right), 183$ (13), 177 (12), 165 (17), 159 (10), 156 (54), 155 (100), 154 (22), 149 (14), 146 (52), 145 (66); TLC R 0.35 (EtOAc/hexane, 1/60).

\section{(2R,5S) 2-Isopropyl-5-methyl-cyclohexyl 2,2-Dimethylpropanoate ${ }^{15}$}

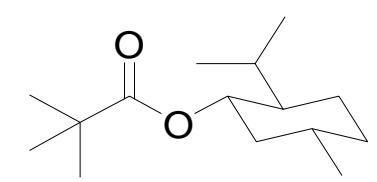

Data: ${ }^{1} \mathrm{H}$ NMR $\left(\mathrm{CDCl}_{3}, 200 \mathrm{MHz}\right) 4.61(\mathrm{dt}, J=10.8,4.4,1 \mathrm{H}), 2.20-1.78$ (m, 2H), 1.74-1.58 (m, 2H), 1.58-1.29 (m, 2H), 1.17 (s, 9H), 1.15-0.98 $(\mathrm{m}, 1 \mathrm{H}), 0.98-0.92(\mathrm{~m}, 1 \mathrm{H}), 0.88(\mathrm{~d}, J=7.6,6 \mathrm{H}), 0.84-0.78(\mathrm{~m}, 1 \mathrm{H}), 0.73$ $(\mathrm{d}, J=7.0,3 \mathrm{H}),{ }^{13} \mathrm{C} \mathrm{NMR}\left(\mathrm{CDCl}_{3}, 50 \mathrm{MHz}\right)$ 178.02, 73.66, 46.99, 40.62, 34.25, 31.23, 27.02, 26.02, 23.17, 21.91, 20.70, 15.95; MS (70 eV) $240\left(\mathrm{M}^{+}, 12\right), 225$ (61), 218 (12), 197 (14), 183 (74), 165 (15), 157 (15), 156 (77), 155 (100), 154 (55), 148 (13); TLC $\mathrm{R}_{f} 0.4$ (EtOAc/hexane, $1 / 60)$.

Acetic acid 17 -(1,5 -dimethyl-hexyl ) -10,13-dimethyl-2, 3, 4, 7, 8, 9, 10, 11, 12, 13, 14, 15, 16, 17-tetradecahydro-1H-cyclopenta[a]phenanthren-3-yl ester ${ }^{16}$

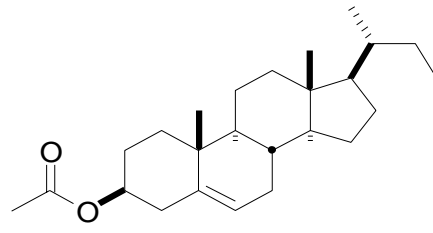

Data: ${ }^{1} \mathrm{H}$ NMR (400 MHz, $\left.\mathrm{CDCl}_{3}\right) 5.35(\mathrm{~d}, J=4.4,1 \mathrm{H}), 4.62-4.54$ (m, 1H), $2.30(\mathrm{~d}, J=7.2,2 \mathrm{H}), 2.00(\mathrm{~s}, 3 \mathrm{H}), 1.99-1.77(\mathrm{~m}, 6 \mathrm{H})$, 1.66-1.04 (m, 20H), $1.00(\mathrm{~s}, 3 \mathrm{H}), 0.90(\mathrm{~d}, J=6.4,3 \mathrm{H}), 0.85(\mathrm{~d}, J=$ $6.8,3 \mathrm{H}), 0.84(\mathrm{~d}, J=6.8,3 \mathrm{H}), 0.66(\mathrm{~s}, 3 \mathrm{H}) ;{ }^{13} \mathrm{C} \mathrm{NMR}(100 \mathrm{MHz}$, $\left.\mathrm{CDCl}_{3}\right)$ 170.31, 139.54, 122.55, 73.87, 56.63, 56.10, 49.98, 42.24, 39.68, 39.47, 38.07, 36.95, 
$36.51,36.14,35.75,31.83,31.80,28.18,27.94,27.71,24.22$, 23.80, 22.76, 22.51, 21.32, 20.98, 19.24, 18.67, 11.79; IR ( $\left.\mathrm{CH}_{2} \mathrm{Cl}_{2}\right) 3060$ (s), 2984 (s), 2305 (m), 1727 (s), 1469 (s), 1420 (s), 1375 (s), 1267 (s), 1137 (m), 1033 (s), 894 (s); MS (70 eV) 428 (M+ , 5), 368 (100), 352 (18), 247 (13), 147 (20); TLC R 0.57 (EtOAc/hexane, 1/9).

2,2-Dimethyl-propanoic acid 17- (1,5-dimethyl-hexyl)-10,13-dimethyl-2,3,4,7,8,9,10, 11,12,13,14,15,16,17-tetradecahydro-1H-cyclopenta[a]phenanthren-3-yl ester

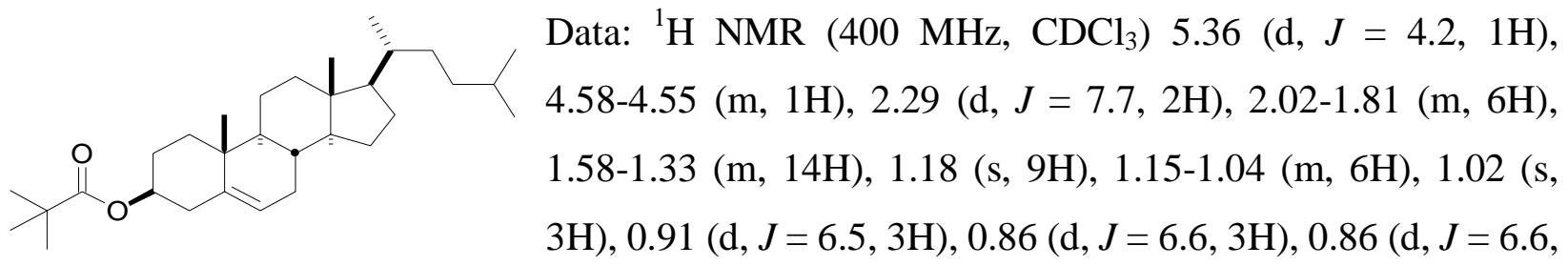

3H), 0.68 (s, 3H); ${ }^{13} \mathrm{C}$ NMR (100 MHz, $\left.\mathrm{CDCl}_{3}\right)$ 177.94, 139.79, 122.47, 73.53, 56.70, 56.16, 50.04, 42.32, 39.75, 39.52, 38.59, 38.02, 37.00, 36.61, 36.20, 35.80, 28.22, 28.00, 27.67, 27.15, 24.28, 23.84, 22.81, 22.55, 21.05, 19.35, 18.72, 11.85; IR $\left(\mathrm{CH}_{2} \mathrm{Cl}_{2}\right) 3057$ (s), 2987 (s), 2305 (m), 1715 (s), 1420 (s), 1263 (s), 1169 (m), 896 (s); MS (70 eV) $470\left(\mathrm{M}^{+}, 5\right), 368$ (100), 352 (18), 260 (14), 147 (19), 145 (15), 81 (13), 57 (20); TLC R 0.67 (EtOAc/hexane, 1/9).

Benzoic acid 17-(1,5-dimethyl-hexyl)-10,13-dimethyl-2,3,4,7,8,9,10,11,12,13,14,15, 16,17tetradecahydro-1H-cyclopenta[a]phenanthren-3-yl

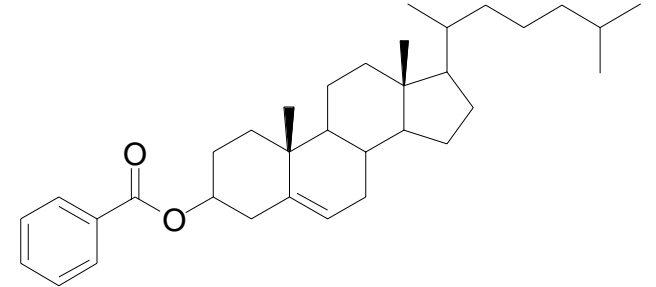
ester

Data: ${ }^{1} \mathrm{H}$ NMR $\left(400 \mathrm{MHz}, \mathrm{CDCl}_{3}\right) 8.05(\mathrm{~d}, J=7.6,2 \mathrm{H})$, 7.56-7.41 (m, 3H), $5.42(\mathrm{~d}, J=4.0,1 \mathrm{H}), 4.91-4.83(\mathrm{~m}$, $1 \mathrm{H}), 2.47(\mathrm{~d}, J=7.6,2 \mathrm{H}), 2.04-0.99$ (m, 29H), 0.93 (d, $J=$ $6.4,3 \mathrm{H}), 0.87(\mathrm{~d}, J=6.8,3 \mathrm{H}), 0.87(\mathrm{~d}, J=6.8,3 \mathrm{H}), 0.70(\mathrm{~s}, 3 \mathrm{H}) ;{ }^{13} \mathrm{C} \mathrm{NMR}\left(100 \mathrm{MHz}, \mathrm{CDCl}_{3}\right)$ $165.99,139.68,132.68,130.88,129.53,128.24,122.77,74.59,56.72,56.18,50.08,42.34,39.77$, 39.53, 38.23, 37.06, 36.67, 36.20, 35.80, 28.23, 28.01, 27.90, 24.30, 23.84, 22.80, 22.56, 21.07, 19.37, 18.73, 11.87; IR ( $\left.\mathrm{CH}_{2} \mathrm{Cl}_{2}\right) 3051$ (s), 2985 (s), 2306 (m), 1711 (m), 1603 (w), 1423 (s), 1279 (s), 1263 (s), 1119 (m), 896 (s); MS (70 eV) 490 (M+, 5), 368 (100), 353 (15), 260 (14), 147 (14), 105 (20); TLC R 0.62 (EtOAc/hexane, 1/9).

\section{General procedure for acylation of tert-butanol and trityl alcohol}


In a dry 50-mL, two-necked, round-bottomed flask was placed $\mathrm{MoO}_{2} \mathrm{Cl}_{2}(19 \mathrm{mg}, 0.1 \mathrm{mmol})$ in $5 \mathrm{~mL}$ of anhydrous toluene. A solution of anhydride $(15 \mathrm{mmol})$ and a given $3^{\circ}$ alcohol $(10 \mathrm{mmol})$ in toluene $(5 \mathrm{~mL})$ was slowly added to the above solution and the reaction mixture was stirred for $30 \mathrm{~min}$ to 1 hour. To the above solution, diisopropylethylamine (1750 $\mu \mathrm{L}, 10 \mathrm{mmol})$ was slowly added at ambient temperature. This mixture was refluxed for 1 to 24 hours. After completion of the reaction as monitored by TLC (for the benzoylation of tryl alcohol), the reaction mixture was quenched with cold, saturated aqueous $\mathrm{NaHCO}_{3}$ solution $(50 \mathrm{~mL})$ at room temperature. The separated organic layer was washed with brine, dried $\left(\mathrm{MgSO}_{4}\right)$, filtered, and evaporated. The crude product was purified by distillation. The product obtained was characterized by routine spectroscopic methods.

\section{Acetic acid tert-butyl ester ${ }^{17}$}

Data: ${ }^{1} \mathrm{H}$ NMR $\left(400 \mathrm{MHz}, \mathrm{CDCl}_{3}\right) 1.95(\mathrm{~s}, 3 \mathrm{H}), 1.43(\mathrm{~s}, 9 \mathrm{H}) ;{ }^{13} \mathrm{C} \mathrm{NMR}(100 \mathrm{MHz}$,
$\left.\mathrm{CDCl}_{3}\right) 170.53,80.12,28.03,22.49$

\section{2, 2-Dimethyl-propionic acid tert-butyl ester ${ }^{18}$}

Data: ${ }^{1} \mathrm{H}$ NMR $\left(400 \mathrm{MHz}, \mathrm{CDCl}_{3}\right) 1.43(\mathrm{~s}, 9 \mathrm{H}), 1.15(\mathrm{~s}, 9 \mathrm{H}) ;{ }^{13} \mathrm{C}$ NMR $(100$
$\left.\mathrm{MHz}, \mathrm{CDCl}_{3}\right) 177.95,79.46,39.14,27.92,27.13$.

\section{Benzoic acid tert-butyl ester ${ }^{19}$}

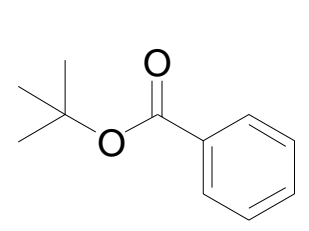

Data: ${ }^{1} \mathrm{H}$ NMR (400 MHz, $\mathrm{CDCl}_{3}$ ) 8.02-7.99 (m, 2H), 7.51-7.38 (m, 3H), 1.60 (s, 9H); ${ }^{13} \mathrm{C} \mathrm{NMR}\left(100 \mathrm{MHz}, \mathrm{CDCl}_{3}\right) 165.65,132.30,131.94,129.31,128.07$, 80.78, 28.09; TLC R 0.61 (EtOAc/hexane, 1/9).

\section{Acetic acid trityl ester ${ }^{20}$}

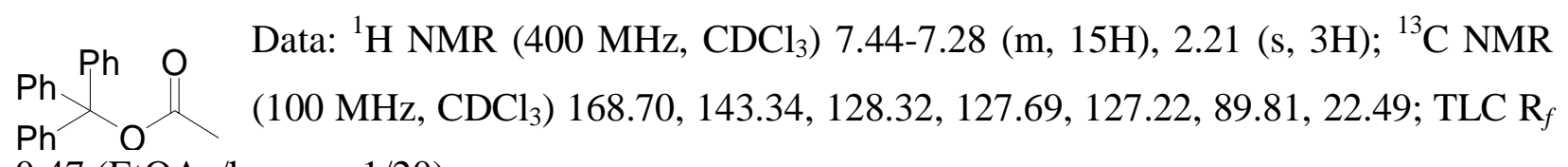
0.47 (EtOAc/hexane, 1/20). 
2, 2-Dimethyl-propionic acid trityl ester ${ }^{21}$

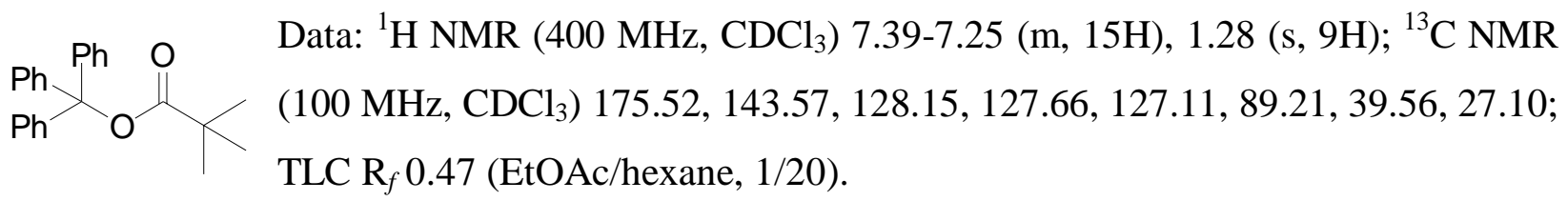

Benzoic acid trityl ester ${ }^{22}$

$\mathrm{Ph} P \mathrm{Ph} \quad \mathrm{O} \quad$ Data: ${ }^{1} \mathrm{H}$ NMR (400 MHz, $\left.\mathrm{CDCl}_{3}\right)$ 8.15-8.13 (m, 2H), 7.58-7.45 (m, $\left.9 \mathrm{H}\right)$, $\mathrm{Ph} \mathrm{O} 7.35-7.25(\mathrm{~m}, 9 \mathrm{H}) ;{ }^{13} \mathrm{C} \mathrm{NMR}\left(100 \mathrm{MHz}, \mathrm{CDCl}_{3}\right)$ 164.41, 143.39, 132.96, $131.28,129.80,128.41,128.37,127.80,127.31,90.50 ;$ TLC $\mathrm{R}_{f} 0.41$ (EtOAc/hexane, 1/20).

trans-11-Acetoxy-10,11-dihydro-5H-dibenzo[a,d]cyclohepten-10-yl Acetate ${ }^{23}$

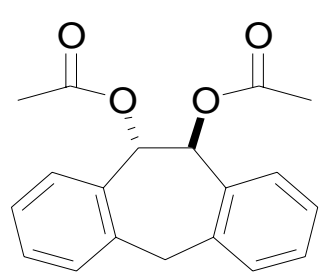

Data: ${ }^{1} \mathrm{H}$ NMR (200 MHz, $\left.\mathrm{CDCl}_{3}\right)$ 7.32-7.20 (m, 8H), 6.53 (s, 2H), 4.18 (s, 2H), 2.16 (s, 6H); ${ }^{13} \mathrm{C}$ NMR (100 MHz, $\left.\mathrm{CDCl}_{3}\right)$ 171.41, 138.22, 137.58, 128.56, 127.98, 127.71, 127.02, 75.24, 40.8, 22.18; $\mathrm{TLC} \quad \mathrm{R}_{f} 0.32$ (EtOAc/hexane, 1/9); Anal. Calcd. For $\mathrm{C}_{19} \mathrm{H}_{18} \mathrm{O}_{4}$ (310.33): C, 73.53; H, 5.85, Found: C, 73.47; H, 5.28.

trans-11-(2,2-Dimethyl-propanoyloxy)-10,11-dihydro-5H-dibenzo[a,d]cyclohepten-10-yl 2,2-Dimethylpropanoate

Data: ${ }^{1} \mathrm{H}$ NMR (200 MHz, $\left.\mathrm{CDCl}_{3}\right)$ 7.27-7.14 (m, 8H), 6.54 (s, 2H), 4.18 (s, 2H), 1.23 (s, 18H); ${ }^{13} \mathrm{C}$ NMR (100 MHz, $\left.\mathrm{CDCl}_{3}\right)$ 178.60, 140.34, 135.23, 129.21, 128.90,

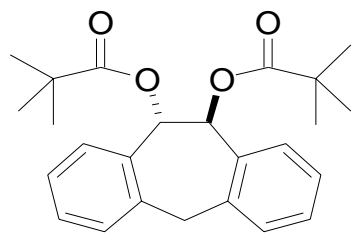
128.81, 127.52, 73.62, 40.09, 38.39, 26.55; MS (70 eV) 394 (2), 293 (49), 190 (27), 178 (35), 137 (37); TLC R 0.58 (EtOAc/hexane, 1/9); Anal. Calcd. for $\mathrm{C}_{25} \mathrm{H}_{30} \mathrm{O}_{4}$ (394.49): C, 76.11; H, 7.66. Found: C, 76.04; H, 7.71.

\section{Naphthalen-2-yl Acetate Ac $^{24}$}

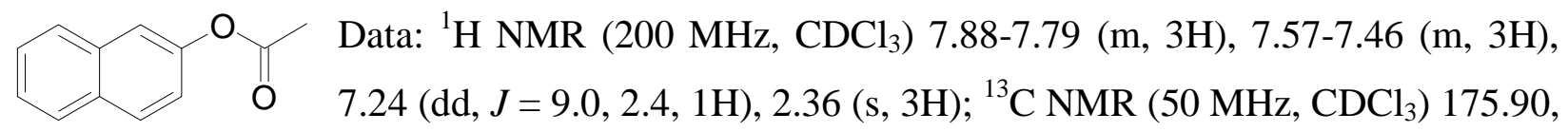
$148.41,133.82$, 131.52, 129.45, 127.81, 127.69, 126.59, 125.74, 121.16, 118.55, 21.10; TLC R 0.39 (EtOAc/hexane, 1/4). 
Naphthalen-2-yl 2, 2-Dimethylpropanoate ${ }^{25}$

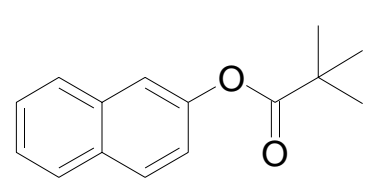

Data: ${ }^{1} \mathrm{H}$ NMR (200 MHz, $\left.\mathrm{CDCl}_{3}\right)$ 7.89-7.80 (m, 3H), 7.57-7.46 (m, 3H), $7.23(\mathrm{dd}, J=9.0,2.4,1 \mathrm{H}), 1.44(\mathrm{~s}, 9 \mathrm{H}) ;{ }^{13} \mathrm{C} \mathrm{NMR}\left(50 \mathrm{MHz}, \mathrm{CDCl}_{3}\right)$ $177.30,148.85,133.85,131.42,129.31,127.78,127.58,126.50,125.56$, 121.18, 118.39, 39.05, 27.10; TLC R 0.54 (EtOAc/hexane, 1/ 4).

2-Naphthyl benzoate ${ }^{26}$

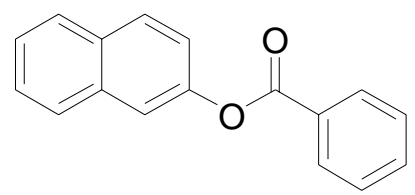

Data: ${ }^{1} \mathrm{H}$ NMR $\left(200 \mathrm{MHz}, \mathrm{CDCl}_{3}\right)$ 8.29-8.24 (m, 2H), 7.92 (d, $J=8.6$, $1 \mathrm{H}), 7.94-7.82(\mathrm{~m}, 2 \mathrm{H}), 7.71-7.48(\mathrm{~m}, 6 \mathrm{H}), 7.38(\mathrm{dd}, J=8.8,2.3,1 \mathrm{H})$;

${ }^{13} \mathrm{C}$ NMR (50 MHz, $\left.\mathrm{CDCl}_{3}\right)$ 165.42, 148.66, 133.85, 133.67, 131.54, $130.24,129.59,129.50,128.63,127.83,127.71,126.60,125.75,121.26,118.71 ; \mathrm{MS}(20 \mathrm{eV}) 248$ (M+, 35), 115 (18), 105 (100), 77 (27); TLC R 0.65 (EtOAc/hexane, 1/ 4).

\section{N-Naphthalen-2-yl-acetamide ${ }^{27}$}

Data: ${ }^{1} \mathrm{H}$ NMR $\left(200 \mathrm{MHz} \mathrm{CDCl}_{3}\right) 8.17(\mathrm{bd}, J=1.4,1 \mathrm{H}), 8.06(\mathrm{bs}, 1 \mathrm{H})$,
$\mathrm{O}$ 125.01, 120.03, 116.81, 24.47; TLC R 0.29 (EtOAc/hexane, 1/4).

\section{$N$-Naphthalen-2-yl-2, 2-dimethyl-propanamide ${ }^{28}$}

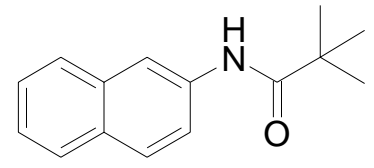

Data: ${ }^{1} \mathrm{H}$ NMR (200 MHz, $\left.\mathrm{CDCl}_{3}\right) 8.27$ (d, $\left.J=2.2,1 \mathrm{H}\right), 7.81-7.77(\mathrm{~m}, 3 \mathrm{H})$, 7.49-7.39 (m, 4H), $1.36(\mathrm{~s}, 9 \mathrm{H}) ;{ }^{13} \mathrm{C} \mathrm{NMR}\left(50 \mathrm{MHz}, \mathrm{CDCl}_{3}\right)$ 176.91, $135.44,133.93,130.63,128.69$, 127.69, 126.54, 126.51, 124.97, 120.01, 116.74, 39.64, 27.58; TLC R 0.26 (EtOAc/hexane, 1/4).

\section{N-2-Napthalenyl-benzamide ${ }^{29}$}

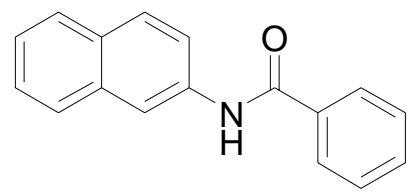

Data: ${ }^{1} \mathrm{H}$ NMR (200 MHz, $\left.\mathrm{CDCl}_{3}\right) 8.35(\mathrm{~d}, J=2.2,1 \mathrm{H}), 7.98-7.78(\mathrm{~m}$, $6 \mathrm{H}), 7.62-7.42(\mathrm{~m}, 6 \mathrm{H}) ;{ }^{13} \mathrm{C} \mathrm{NMR}\left(50 \mathrm{MHz}, \mathrm{CDCl}_{3}\right)$ 166.05, 135.43, 135.02, 133.93, 131.95, 130.86, 128.87, 128.51, 127.78, 127.63, 127.11, 
126.60, 125.19, 120.18, 117.16; MS (20 eV) $247\left(\mathrm{M}^{+}, 86\right), 115$ (20), 105 (100), 77 (37); TLC $\mathrm{R}_{f}$ 0.24 (EtOAc/hexane, 1/4).

Naphthalen-2-yl-carbamic acid tert-butyl ester ${ }^{30}$

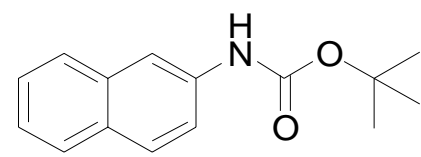

Data: ${ }^{1} \mathrm{H}$ NMR $\left(200 \mathrm{MHz}, \mathrm{CD}_{2} \mathrm{Cl}_{2}\right) 8.26(\mathrm{~d}, J=2.0,1 \mathrm{H}), 7.80-7.76(\mathrm{~m}$, $3 \mathrm{H})$ 7.47-7.40 (m, 4H), 1.37 (s, 9H); ${ }^{13} \mathrm{C}$ NMR (100 MHz, $\left.\mathrm{CD}_{2} \mathrm{Cl}_{2}\right)$ $176.77,135.42$, 133.86, 130.58, 128.58, 127.60, 127.46, 126.41, 124.88, 120.03, 116.74, 39.66, 27.62; $\mathrm{TLC} \mathrm{R}_{f} 0.30$ (EtOAc/hexane, 1/4).

\section{$S$-Naphthalen-2-yl Thioacetate ${ }^{31}$}

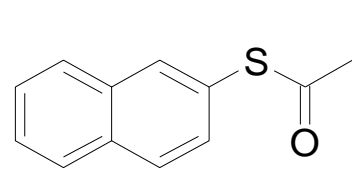

Data: ${ }^{1} \mathrm{H}$ NMR (200 MHz, $\left.\mathrm{CDCl}_{3}\right)$ 7.97-7.82 (m, 4H), 7.58-7.45 (m, 3H), 2.47 (s, 3H); ${ }^{13} \mathrm{C}$ NMR (50 MHz, $\left.\mathrm{CDCl}_{3}\right)$ 194.43, 134.35, 133.55, 133.36, $130.91,128.84,128.01,127.81,127.19,126.60,125.26,30.18$; TLC $\mathrm{R}_{f}$

0.42 (EtOAc/hexane, 1/20).

\section{$S$-Naphthalen-2-yl 2, 2-Dimethyl-thiopropanoate ${ }^{32}$}

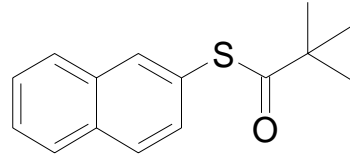

Data: ${ }^{1} \mathrm{H}$ NMR $\left(200 \mathrm{MHz}, \mathrm{CDCl}_{3}\right) 7.91(\mathrm{dd}, J=10.0,1.4,1 \mathrm{H}), 7.88-7.82$ (m, 3H), 7.54-7.49 (m, 2H), $7.43(\mathrm{dd}, J=8.6,1.8,1 \mathrm{H}), 1.36(\mathrm{~s}, 9 \mathrm{H}) ;{ }^{13} \mathrm{C}$ NMR (50 MHz, $\mathrm{CDCl}_{3}$ ) 204.98, 134.79, 133.65, 133.29, 131.50, 128.65, 127.95, 127.81, 127.01, 126.46, 125.51, 46.96, 27.37; TLC $\mathrm{R}_{f} 0.46$ (EtOAc/hexane, 1/40).

\section{$S$-[2]-Naphthyl thiobenzoate ${ }^{37}$}

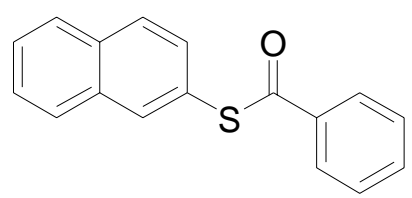

Data: ${ }^{1} \mathrm{H}$ NMR (200 MHz, $\left.\mathrm{CDCl}_{3}\right)$ 8.10-8.05 (m, 3H), 7.95-7.84 (m, 3H), 7.64-7.46 (m, 6H); ${ }^{13} \mathrm{C}$ NMR (50 MHz, $\mathrm{CDCl}_{3}$ ) 190.47, 136.73, $135.05,133.76,133.70,133.50,131.45,128.87,128.83,128.04,127.87$, 127.57, 127.22, 126.60, 124.73; MS (20 eV) $264\left(\mathrm{M}^{+}, 18\right), 115$ (17), 105 (100), 77 (24); TLC $\mathrm{R}_{f}$ 0.40 (EtOAc/hexane, 1/40).

\section{$N$-Benzyl-acetamide ${ }^{33}$}

Data: ${ }^{1} \mathrm{H}$ NMR (400 MHz, $\left.\mathrm{CDCl}_{3}\right)$ 7.33-7.24 (m, 5H), 6.11 (s, 1H), 4.38 (d, $J=$ 5.7, 2H), 1.98 (s, 3H); ${ }^{13} \mathrm{C}$ NMR (100 MHz, $\left.\mathrm{CDCl}_{3}\right)$ 170.05, 138.21, 128.55, 
127.68, 127.31, 43.56, 23.01; MS (ESI) $172\left(\mathrm{M}+\mathrm{Na}^{+}, 62\right), 155$ (31); TLC R 0.43 (EtOAc/hexane, $1 / 1)$.

$N$-Benzyl-2, 2-dimethyl-propionamide ${ }^{34}$

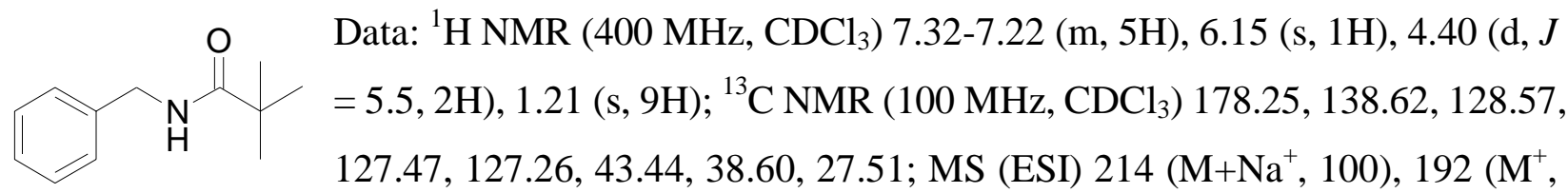

15), 185 (10); TLC R 0.20 (EtOAc/hexane, 1/10).

\section{$N$-Benzyl-benzamide ${ }^{35}$}

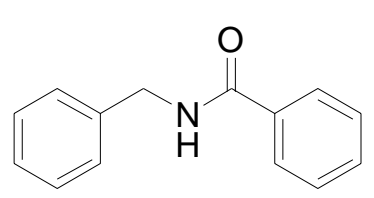

Data: ${ }^{1} \mathrm{H}$ NMR $\left(400 \mathrm{MHz}, \mathrm{CDCl}_{3}\right) 7.79(\mathrm{dd}, J=8.0,1.4,2 \mathrm{H}), 7.50(\mathrm{t}, J=$ $7.4,1 \mathrm{H}), 7.45-7.30(\mathrm{~m}, 7 \mathrm{H}), 6.44(\mathrm{bs}, 1 \mathrm{H}), 4.65(\mathrm{~d}, J=5.6,2 \mathrm{H}) ;{ }^{13} \mathrm{C} \mathrm{NMR}$ $\left(100 \mathrm{MHz}, \mathrm{CDCl}_{3}\right)$ 167.31, 138.20, 134.43, 131.52, 128.79, 128.59, 127.92, 127.63, 126.94, 44.15; MS (20 eV) $211\left(\mathrm{M}^{+}, 75\right), 105$ (100), 77 (51), 51 (14); TLC R 0.40 (EtOAc/hexane, 1/10).

\section{Benzyl-carbamic acid tert-butyl ester ${ }^{36}$}

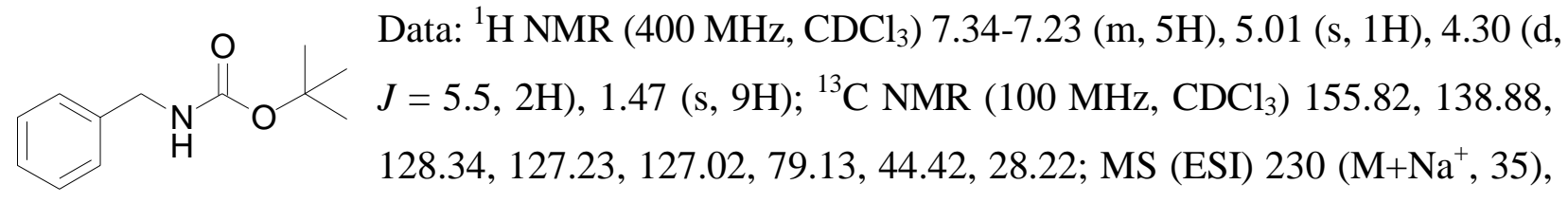
181 (13); TLC R 0.43 (EtOAc/hexane, 1/10).

\section{$N, N$-Diisopropyl-acetamide ${ }^{37}$}

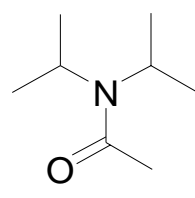

Data: ${ }^{1} \mathrm{H}$ NMR (200 MHz, $\left.\mathrm{CDCl}_{3}\right)$ 3.92-3.82 (m, 1H), 3.56-3.49 (m, 1H), 2.07 (s, $3 \mathrm{H}), 1.35(\mathrm{~d}, J=6.4,6 \mathrm{H}), 1.19(\mathrm{~d}, J=6.6,6 \mathrm{H}) ;{ }^{13} \mathrm{C} \mathrm{NMR}\left(50 \mathrm{MHz}, \mathrm{CDCl}_{3}\right) 169.66$, 48.98, 45.15, 23.23, 20.41, 20.11; MS (20 eV) $143\left(\mathrm{M}^{+}, 13\right), 135$ (17), 134 (11), 117 (22), 104 (100); TLC R 0.31 (EtOAc/hexane, 1/20).

\section{$N$-tert-Butyl-acetamide ${ }^{38}$}

O Data: ${ }^{1} \mathrm{H}$ NMR $\left(400 \mathrm{MHz}, \mathrm{CDCl}_{3}\right) 5.33$ (bs, $\left.1 \mathrm{H}\right), 1.90(\mathrm{~s}, 3 \mathrm{H}), 1.33(\mathrm{~s}, 9 \mathrm{H}) ;{ }^{13} \mathrm{C}$ NMR N $\left(100 \mathrm{MHz}, \mathrm{CDCl}_{3}\right) 169.42,51.11,28.75,24.51$; TLC R $\mathrm{R}_{f} 0.5$ (EtOAc/hexane, 1/4). 
$N$-tert-Butyl-2,2-dimethyl-propionamide ${ }^{39}$

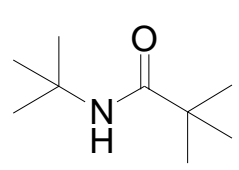

Data: ${ }^{1} \mathrm{H}$ NMR (400 MHz, $\left.\mathrm{CDCl}_{3}\right) 5.38$ (bs, 1H), 1.32 (s, 9H), 1.14 (s, 9H); ${ }^{13} \mathrm{C}$ NMR (100 MHz, $\left.\mathrm{CDCl}_{3}\right)$ 177.77, 50.64, 38.94, 28.71, 27.64; TLC $\mathrm{R}_{f} 0.45$ (EtOAc/hexane, 1/4).

\section{$N$-tert-Butyl-benzamide ${ }^{40}$}

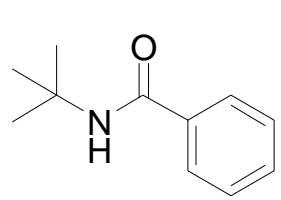

Data: ${ }^{1} \mathrm{H}$ NMR (400 MHz, $\left.\mathrm{CDCl}_{3}\right) 7.71(\mathrm{dd}, J=8.4,1.3,2 \mathrm{H}), 7.47-7.37(\mathrm{~m}, 3 \mathrm{H})$, 5.97 (bs, 1H), 1.47 (s, 9H); ${ }^{13} \mathrm{C}$ NMR (100 MHz, $\left.\mathrm{CDCl}_{3}\right)$ 166.85, 135.92, 130.99, 128.40, 126.65, 51.53, 28.83; TLC R $\mathrm{R}_{f} 0.25$ (EtOAc/hexane, 1/4).

tert-Butyl-carbamic acid-tert-butyl ester ${ }^{41}$

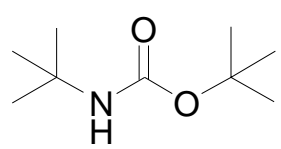

Data: ${ }^{1} \mathrm{H}$ NMR (400 MHz, $\left.\mathrm{CDCl}_{3}\right) 4.45$ (bs, 1H), 1.42 (s, 9H), 1.28 (s, 9H); ${ }^{13} \mathrm{C}$ NMR (100 MHz, $\left.\mathrm{CDCl}_{3}\right)$ 154.44, 78.43, 49.84, 28.93, 28.36; TLC $\mathrm{R}_{f} 0.40$ (EtOAc/hexane, 1/4).

\section{Thioacetic acid $S$-benzyl ester ${ }^{42}$}

Data: ${ }^{1} \mathrm{H}$ NMR $\left(400 \mathrm{MHz}, \mathrm{CDCl}_{3}\right)$ 7.31-7.26 (m, 5H), 4.13 (s, 2H), 2.36 (s, 3H);

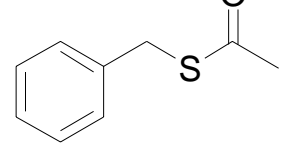

${ }^{13} \mathrm{C}$ NMR $\left(100 \mathrm{MHz}, \mathrm{CDCl}_{3}\right)$ 195.05, 137.57, 128.78, 128.60, 127.24, 33.44, 30.27; MS (20 eV) 167 (M+1 $\left.{ }^{+}, 27\right), 123$ (19), 91 (100), 77 (10), 65 (17); TLC R

0.2 (hexane).

\section{2, 2-Dimethyl-thiopropionic acid $S$-benzyl ester ${ }^{43}$}

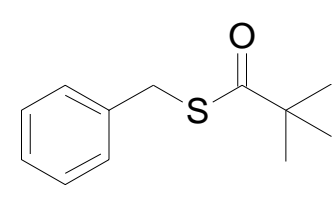

Data: ${ }^{1} \mathrm{H}$ NMR (400 MHz, $\mathrm{CDCl}_{3}$ ) 7.33-7.25 (m, 5H), 4.13 (s, 2H), 1.30 (s, 9H); $\left.{ }^{13} \mathrm{C} \mathrm{NMR} \mathrm{(100} \mathrm{MHz,} \mathrm{CDCl}_{3}\right)$ 205.65, 137.64, 128.66, 128.39, 126.95 , 46.17, 32.84, 27.22; MS (20 eV) 209 (M+1 $\left.1^{+}, 100\right), 91$ (92), 85 (100), 65 (18),

57 (100); TLC $\mathrm{R}_{f} 0.6$ (hexane).

\section{$S$-Benzyl-thiobenzoate ${ }^{44}$}

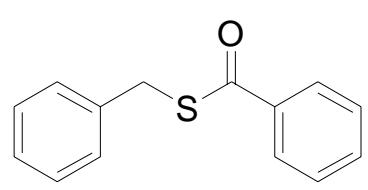

Data: ${ }^{1} \mathrm{H}$ NMR (400 MHz, $\left.\mathrm{CDCl}_{3}\right) 7.98(\mathrm{dd}, J=8.4,1.3,2 \mathrm{H}), 7.57(\mathrm{t}, J=$ 7.4, 1H), 7.47-7.24 (m, 7H), 4.33 (s, $2 \mathrm{H}) ;{ }^{13} \mathrm{C}$ NMR (100 MHz, $\mathrm{CDCl}_{3}$ ) 191.26, 137.46, 136.82, 133.40, 128.96, 128.63, 128.60, 127.30, 127.28, 33.33; MS (20 eV) $228\left(\mathrm{M}^{+}, 18\right), 65$ (55), 105 (100), 91 (60), 77 (27); TLC $\mathrm{R}_{f} 0.46$ 
(EtOAc/hexane, 1/20).

S-tert-butyl Thioacetate ${ }^{45}$

O Data: ${ }^{1} \mathrm{H}$ NMR $\left(200 \mathrm{MHz}, \mathrm{CDCl}_{3}\right) 2.26(\mathrm{~s}, 3 \mathrm{H}), 1.43$ (s, 9H); ${ }^{13} \mathrm{C}$ NMR (50 MHz, $\mathrm{S} \mathrm{CDCl}_{3}$ ) 195.53, 47.17, 30.76, 29.27; TLC R 0.33 (hexane).

S-tert-butyl 2, 2-Dimethyl-thiopropanoate ${ }^{46}$

O Data: ${ }^{1} \mathrm{H}$ NMR (200 MHz, $\left.\mathrm{CDCl}_{3}\right) 1.44$ (s, 9H), 1.19 (s, 9H); ${ }^{13} \mathrm{C}$ NMR $(50 \mathrm{MHz}$,

$\left.>\mathrm{CDCl}_{3}\right)$ 207.97, 65.83, 46.96, 29.77, 27.37; MS (70 eV) $174\left(\mathrm{M}^{+}, 10\right), 85(18), 72$ (14), 57 (100); TLC R 0.26 (hexane).

S-tert-butyl thiobenzoate ${ }^{47}$

O Data: ${ }^{1} \mathrm{H}$ NMR $\left(200 \mathrm{MHz}, \mathrm{CDCl}_{3}\right) 7.92(\mathrm{dd}, J=8.4,1.8,2 \mathrm{H}), 7.53-7.38(\mathrm{~m}, 3 \mathrm{H})$, 1.58 (s, 9H); ${ }^{13} \mathrm{C}$ NMR (50 MHz, $\left.\mathrm{CDCl}_{3}\right)$ 192.41, 138.10, 132.74, 128.27, 126.78, 47.73, 29.72; MS (70 eV) $194\left(\mathrm{M}^{+}, 8\right), 138$ (15), 105 (100), (51), 57 (24), 51 (22); TLC R 0.67 (EtOAc/hexane, 1/4). 
On-line text and Table 4' for a complete study regarding the scope of functional group compatibility

Table 4'. Acetylation, pivalation, and chemoselective acylation of functionalized substrates

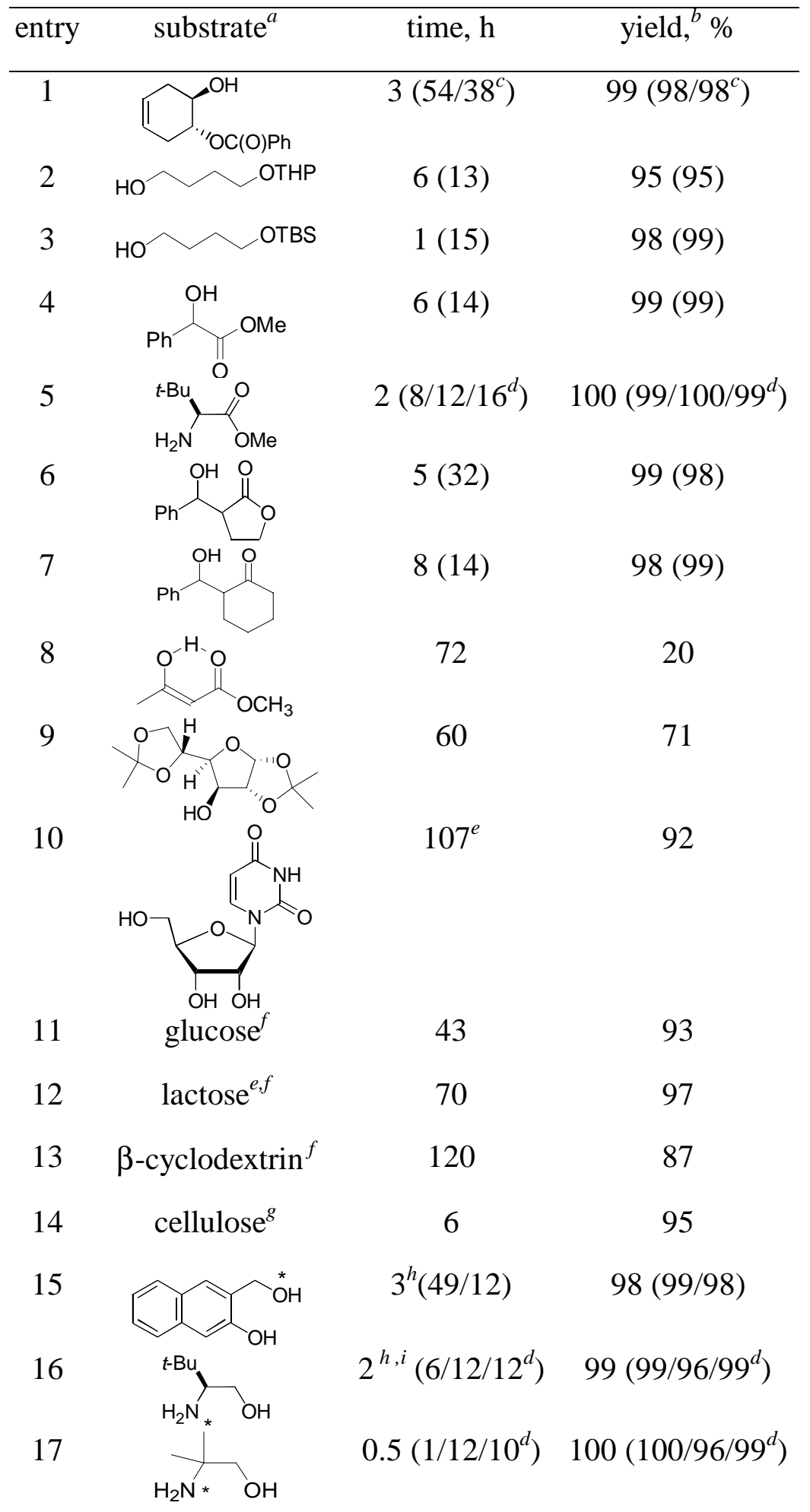


${ }^{a} 1.5$ equiv of anhydride was used unless otherwise stated. ${ }^{b}$ Isolated yields and characterized spectroscopically. ${ }^{c}$ The data in parentheses correspond to pivalation and benzoylation, respectively, unless otherwise stated ${ }^{d}$ The third data in parentheses represent $t$-Boc protection. ${ }^{e}$ Three equiv of anhydride was used. ${ }^{f}$ No solvent was used. ${ }^{g}$ Carried out at 100-110 ${ }^{\circ} \mathrm{C} .{ }^{h}$ Asterisk signifies the reactive site. ${ }^{i}$ For effective mono-acylation, 0.95 equiv of anhydride was used. 


\section{6-Acetoxy-cyclohex-3-enyl benzoate}

Data: ${ }^{1} \mathrm{H}$ NMR $\left(200 \mathrm{MHz}, \mathrm{CDCl}_{3}\right) 8.01(\mathrm{dd}, J=8.4,1.4,2 \mathrm{H}), 7.55-7.40(\mathrm{~m}, 3 \mathrm{H}), 5.63(\mathrm{~d}, J=2.4$, 2H), 5.35-5.25 (m, 2H), 2.70-2.55 (m, 2H), 2.35-2.25 (m, 2H), 1.98 (s, 3H); ${ }^{13} \mathrm{C} \mathrm{NMR} \mathrm{(50} \mathrm{MHz,}$

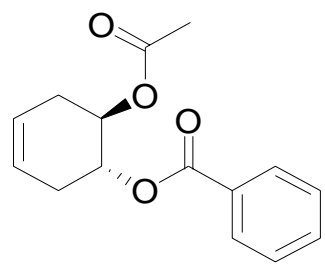
$\left.\mathrm{CDCl}_{3}\right)$ 170.46, 165.89, 133.01, 130.03, 129.57, 128.36, 123.73, 123.67, 70.78, 69.73, 30.03, 20.85; IR $\left(\mathrm{CH}_{2} \mathrm{Cl}_{2}\right) 3052$ (w), 1735 (s), 1720 (s), 1609 (w), 1525 (w), 1316 (m), 1265 (s), 1243 (s), 1119 (m), 1046 (m), 910 (s), 735 (s); MS (70 eV) $261\left(\mathrm{M}+1^{+}, 100\right), 259$ (18), 139 (10), 105 (100), 78 (98); TLC $\mathrm{R}_{f} 0.35$ (EtOAc/hexane, 1/15).

\section{6-(2,2-Dimethyl-propanoyloxy)-cyclohex-3-enyl benzoate}

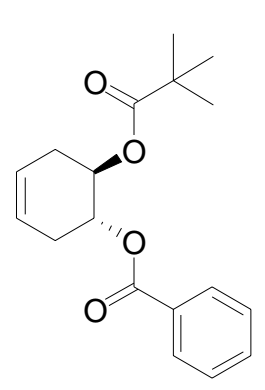

Data: ${ }^{1} \mathrm{H}$ NMR $\left(200 \mathrm{MHz}, \mathrm{CDCl}_{3}\right) 8.01(\mathrm{dd}, J=8.4,1.6,2 \mathrm{H}), 7.55-7.38(\mathrm{~m}, 3 \mathrm{H})$, $5.62(\mathrm{~d}, J=2.4,2 \mathrm{H}), 5.37-5.25(\mathrm{~m}, 2 \mathrm{H}), 2.75-2.55(\mathrm{~m}, 2 \mathrm{H}), 2.40-2.18(\mathrm{~m}, 2 \mathrm{H})$, 1.06 (s, 9H); ${ }^{13} \mathrm{C}$ NMR (50 MHz, $\left.\mathrm{CDCl}_{3}\right)$ 177.96, 165.92, 133.06, 130.06, 129.71, 128.36, 123.84, 123.79, 70.86, 69.65, 38.57, 30.30, 30.16, 26.85; IR $\left(\mathrm{CH}_{2} \mathrm{Cl}_{2}\right)$ 3045 (m), 2985 (s), 1723 (s), 1422 (s), 1282 (s), 1251 (s), 1160 (s), 1117 (s), 1028 (m), 896 (s); MS (70 eV) 303 (M+1 $\left.{ }^{+}, 22\right), 201$ (70), 181 (100), 77 (5); TLC R 0.32 (EtOAc/hexane, 1/15).

\section{trans-4,5-bis-Benzoyloxy-cyclohexene ${ }^{48}$}

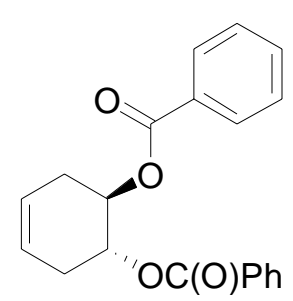

Data: ${ }^{1} \mathrm{H}$ NMR $\left(400 \mathrm{MHz}, \mathrm{CDCl}_{3}\right) 7.98(\mathrm{dd}, J=8.4,1.6,4 \mathrm{H}), 7.52-7.48(\mathrm{~m}$, 2H), 7.39-7.35 (m, 4H), 5.69-5.68 (m, 2H), 5.53-5.50 (m, 2H), 2.81-2.76 (m, 2H), 2.44-2.38 (m, 2H); ${ }^{13} \mathrm{C}$ NMR (100 MHz, $\left.\mathrm{CDCl}_{3}\right)$ 166.01, 132.95, 130.01, 129.60, 128.29, 123.77, 70.66, 30.22; TLC R 0.30 (EtOAc/hexane, 1/20).

\section{Acetic acid 4-(tetrahydro-pyran-2-yloxy)-butyl ester ${ }^{49}$}

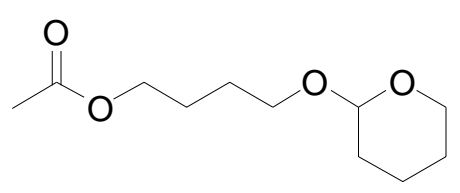

Data: ${ }^{1} \mathrm{H}$ NMR $\left(400 \mathrm{MHz}, \mathrm{CDCl}_{3}\right) 4.44(\mathrm{t}, J=1.6,1 \mathrm{H}), 3.96(\mathrm{t}, J=$ 6.8, 2H), 3.71-3.61 (m, 2H), 3.28-3.25 (m, 2H), $1.91(\mathrm{~s}, 3 \mathrm{H})$, 1.59-1.38 (m, $10 \mathrm{H}) ;{ }^{13} \mathrm{C} \mathrm{NMR}\left(100 \mathrm{MHz}, \mathrm{CDCl}_{3}\right)$ 171.11, 98.82, 66.89, 64.32, 62.28, 30.68, 26.21, 25.56, 25.43, 20.93, 19.57; MS (20 eV) 217 (M+1 , 58), 175 (100), 133 (56), 115 (34), 85 (32); TLC R 0.4 (EtOAc/hexane, 1/10). 


\section{2, 2-Dimethyl-propionic acid 4-(tetrahydro-pyran-2-yloxy)-butyl ester}

Data: ${ }^{1} \mathrm{H}$ NMR $\left(400 \mathrm{MHz}, \mathrm{CDCl}_{3}\right) 4.55(\mathrm{dd}, J=4.4,2.8,1 \mathrm{H}), 4.06(\mathrm{t}, J=6.0,2 \mathrm{H}), 3.86-3.71(\mathrm{~m}$, 2H), 3.50-3.38 (m, 2H), 1.82-1.48 (m, $10 \mathrm{H}), 1.17$ (s, 9H); ${ }^{13} \mathrm{C}$ NMR (100 MHz, $\left.\mathrm{CDCl}_{3}\right)$ 178.34,


$98.67,66.80,64.02,62.13,38.55,30.56,27.03,26.11,25.48$, 25.31, 19.46; IR $\left(\mathrm{CH}_{2} \mathrm{Cl}_{2}\right) 3066$ (s), 2961 (s), 1721 (s), 1442 (s), 1289 (s), 1265 (m), 1245 (s), 1166 (s), 1076 (s), 1034 (s), 973 (s), 900 (s); MS (20 eV) 259 (M+1 $\left.{ }^{+}, 6\right), 175$ (32), 157 (34), 118 (8), 101 (36), 85 (84), 57 (100); TLC $\mathrm{R}_{f} 0.4$ (EtOAc/hexane, 1/4).

\section{Acetic acid 4-(tert-butyl-dimethyl-silanyloxy)-butyl ester ${ }^{503}$}

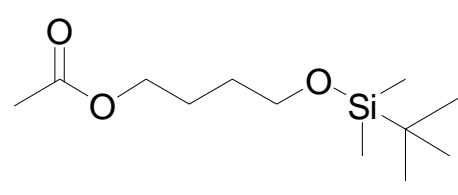

Data: ${ }^{1} \mathrm{H}$ NMR (400 MHz, $\left.\mathrm{CDCl}_{3}\right) 4.03(\mathrm{t}, J=6.5,2 \mathrm{H}), 3.58(\mathrm{t}, J=$ 6.2, 2H), $1.98(\mathrm{~s}, 3 \mathrm{H}), 1.67-1.60(\mathrm{~m}, 2 \mathrm{H}), 1.55-1.48(\mathrm{~m}, 2 \mathrm{H}), 0.84(\mathrm{~s}$, 9H), -0.01 (s, 6H); $\left.{ }^{13} \mathrm{C} \mathrm{NMR} \mathrm{(100} \mathrm{MHz,} \mathrm{CDCl}_{3}\right)$ 170.81, 64.20, 62.39, 29.06, 25.76, 25.09, 20.73, 18.13, -5.51; MS (20 eV) $246\left(\mathrm{M}^{+}, 4\right), 149$ (8), 111 (16), 99 (18), 71 (52), 57 (100); TLC R 0.4 (EtOAc/hexane, 1/10).

\section{2, 2-Dimethyl-propionic acid 4-(tert-butyl-dimethyl-silanyloxy)-butyl ester}

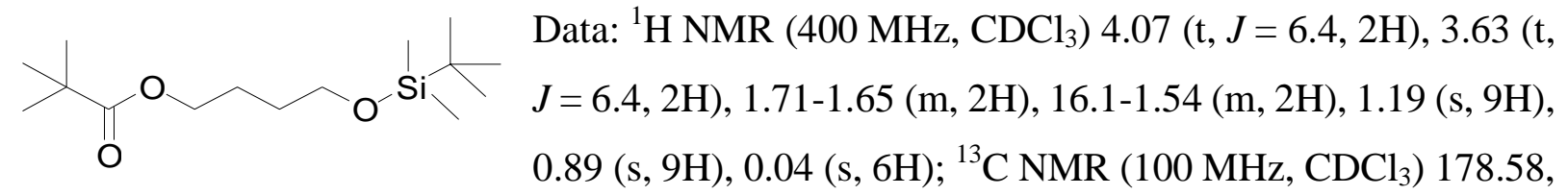
64.23, 62.23, 38.71, 29.28, 27.18, 25.92, 25.26, 18.30, -5.33; IR $\left(\mathrm{CH}_{2} \mathrm{Cl}_{2}\right) 2998$ (w), $2957(\mathrm{~m})$, 2856 (m), 1719 (s), 1602 (w), 1481 (m), 1287 (m), 1266 (m), 1167 (s), 1094 (m), 838 (s); MS (20 eV) $289\left(\mathrm{M}+1^{+}, 100\right), 187$ (16); TLC R 0.42 (EtOAc/hexane, 1/4).

\section{Methyl 2-Acetoxy-2-phenyl-aceate ${ }^{51}$}

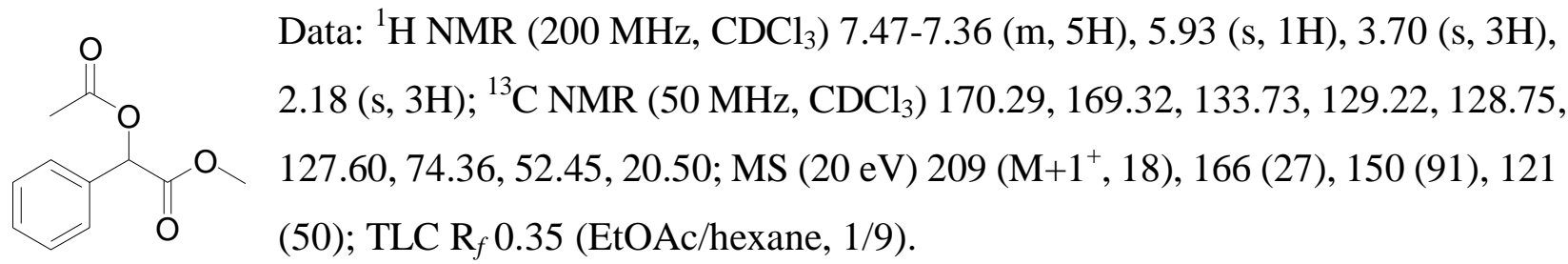

\section{1-Methoxycarbonyl-1-phenyl-methyl 2, 2-Dimethyl-propanoate ${ }^{52}$}


Data: ${ }^{1} \mathrm{H}$ NMR (400 MHz, $\left.\mathrm{CDCl}_{3}\right)$ 7.34-7.33 (m, 5H), $5.18(\mathrm{~d}, J=4.4,1 \mathrm{H}), 3.76$ (s, 3H), 1.25 (s, 9H); $\left.{ }^{13} \mathrm{C} \mathrm{NMR} \mathrm{(100} \mathrm{MHz,} \mathrm{CDCl}_{3}\right)$ 177.9, 169.47, 138.24, 128.60, 128.49, 126.57, 72.88, 52.99, 41.17, 26.49; $\mathbb{R}\left(\mathrm{CH}_{2} \mathrm{Cl}_{2}\right) 2937$ (m), 2856 (m), $1771(\mathrm{~m}), 1742$ (m), 1698 (m), 1595 (m), 1347 (m), 1265 (s), 1233 (w), 740 (s); MS (20 eV) $250\left(\mathrm{M}^{+}, 4\right), 154$ (12), 149 (15), 136 (13), 107 (14); TLC R 0.62 (EtOAc/hexane, $1 / 9)$.

(S)-Methyl 2-Acetylamino-3, 3-dimethyl-butanoate ${ }^{53}$

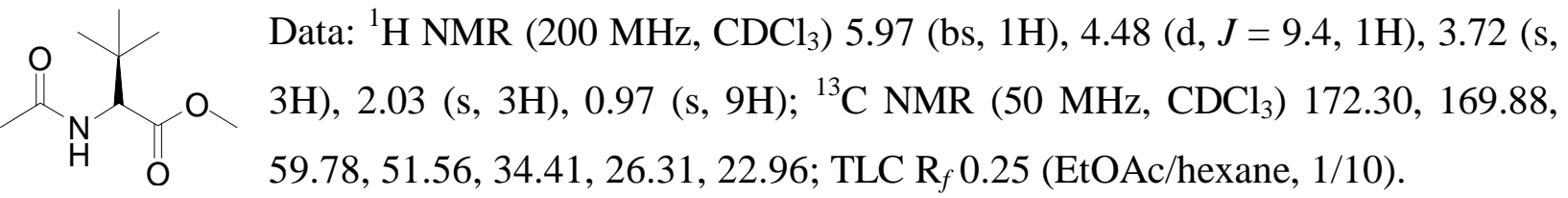

(S)-Methyl 2-(2, 2-Dimethyl-propanoylamino)-3, 3-dimethyl-butanoate

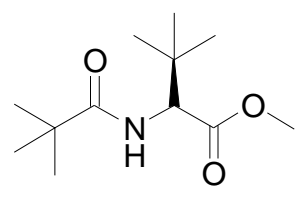

Data: ${ }^{1} \mathrm{H}$ NMR $\left(200 \mathrm{MHz}, \mathrm{CDCl}_{3}\right) 6.14(\mathrm{bd}, J=8.0,1 \mathrm{H}), 4.47$ (d, $\left.J=9.4,1 \mathrm{H}\right)$, 3.72 (s, 3H), 1.22 (s, 9H), 0.96 (s, 9H); ${ }^{13} \mathrm{C}$ NMR (50 MHz, $\left.\mathrm{CDCl}_{3}\right)$ 178.06, 172.49, 59.48, 51.69, 38.81, 34.82, 27.40, 26.45; MS (ESI) 459 (100), 230 $\left(\mathrm{M}+1^{+}, 45\right) ;$ TLC R $\mathrm{R}_{f} 0.23$ (EtOAc/hexane, 1/10).

(2S)-Methyl 2-benzoylamino-3,3-dimethylbutanoate ${ }^{54}$

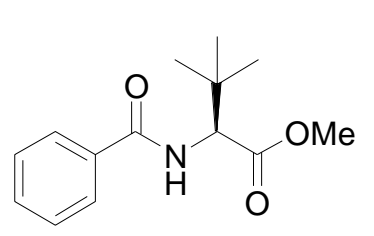

Data: ${ }^{1} \mathrm{H}$ NMR (200 MHz, $\left.\mathrm{CDCl}_{3}\right) 7.80(\mathrm{dd}, J=7.8,1.6,2 \mathrm{H}), 7.56-7.40(\mathrm{~m}$, $3 \mathrm{H}), 6.65(\mathrm{bd}, J=8.2,1 \mathrm{H}), 4.71(\mathrm{~d}, J=9.6,1 \mathrm{H}), 3.76(\mathrm{~s}, 3 \mathrm{H}), 1.05(\mathrm{~s}, 9 \mathrm{H})$;

${ }^{13} \mathrm{C}$ NMR $\left(50 \mathrm{MHz}, \mathrm{CDCl}_{3}\right) 172.16,167.12,134.09,131.62,128.49$, 126.95, 60.05, 51.69, 34.93, 26.40; TLC R 0.35 (EtOAc/hexane, 1/5).

2-tert-Butoxycarbonylamino-3, 3-dimethyl-butyric acid methyl ester ${ }^{55}$

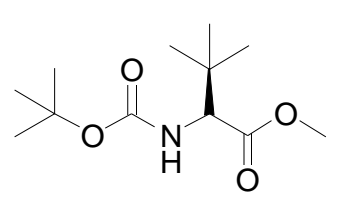

Data: ${ }^{1} \mathrm{H}$ NMR (400 MHz, $\left.\mathrm{CDCl}_{3}\right) 5.09$ (bd, $\left.J=8.4,1 \mathrm{H}\right), 4.10$ (d, $J=9.6$, $1 \mathrm{H}), 3.72(\mathrm{~s}, 3 \mathrm{H}), 1.45$ (s, 9H), 0.98 (s, 9H); ${ }^{13} \mathrm{C} \mathrm{NMR}\left(100 \mathrm{MHz}, \mathrm{CDCl}_{3}\right)$ 172.51, 155.50, 77.33, 61.64, 51.64, 34.63, 28.33, 26.48; MS (ESI) 268 (M+Na $\left.{ }^{+}, 100\right), 246\left(\mathrm{M}^{+}, 10\right), 244$ (50), 146 (21); TLC $\mathrm{R}_{f} 0.23$ (EtOAc/hexane, 1/9). 


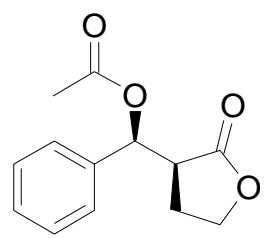

Data: ${ }^{1} \mathrm{H}$ NMR (400 MHz, $\left.\mathrm{CDCl}_{3}\right) 7.32-7.22(\mathrm{~m}, 5 \mathrm{H}), 6.20(\mathrm{~d}, J=3.8,1 \mathrm{H})$, $4.27(\mathrm{dt}, J=8.0,4.0,1 \mathrm{H}), 4.12(\mathrm{dt}, J=8.0,4.0,1 \mathrm{H}), 2.95(\mathrm{dt}, J=12.0,4.0,1 \mathrm{H})$, 2.42-2.37 (m, 1H), $2.08(\mathrm{~s}, 3 \mathrm{H}), 2.10-2.05(\mathrm{~m}, 1 \mathrm{H}) ;{ }^{13} \mathrm{C} \mathrm{NMR}\left(100 \mathrm{MHz}, \mathrm{CDCl}_{3}\right)$ $175.27,169.62,136.77,128.63,128.59,126.78,73.56,66.37,44.35,24.67$, 21.02; IR ( $\left.\mathrm{CH}_{2} \mathrm{Cl}_{2}\right) 3945$ (s), 2987 (s), 2686 (s), 2411 (s), 2306 (s), 1771 (s), 1742 (s), 1422 (s), 1281 (s), 1251 (s), 1163 (m), 1026 (m), 896 (s), 453 (w); MS (20 eV) 234 (M+10), 191 (50), 174 (98), 129 (35), 115 (64); TLC $\mathrm{R}_{f} 0.30$ (ether/hexane, 1/20); HR-MS Calcd. For $\mathrm{C}_{18} \mathrm{H}_{14} \mathrm{O}_{3}$ : 278.0943, found: 278.0943 .

\section{(3-Pivaloyloxy-3-phenyl)methyl-dihydrofuran-2-one (syn isomer)}

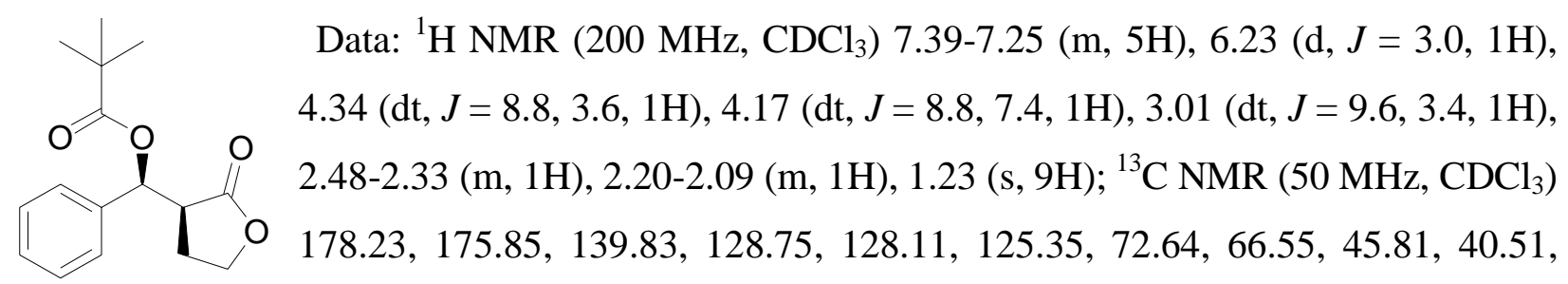
26.93, 23.06; IR ( $\left.\mathrm{CH}_{2} \mathrm{Cl}_{2}\right) 3055$ (m), 2254 (w), 1771 (w), 1742 (w), 1591 (w), 1373 (w), 1270 (m), 909 (s), 720 (m), 651 (m); MS (70 ev) $276\left(\mathrm{M}^{+}, 5\right), 191$ (100), 174 (48), 131 (20), 115 (48), 105 (20), 91 (20), 57 (68); TLC R 0.33 (ether/hexane, 1/20).

\section{(2-Oxo-cyclohexyl)-phenyl-methyl Acetate ${ }^{56}$}

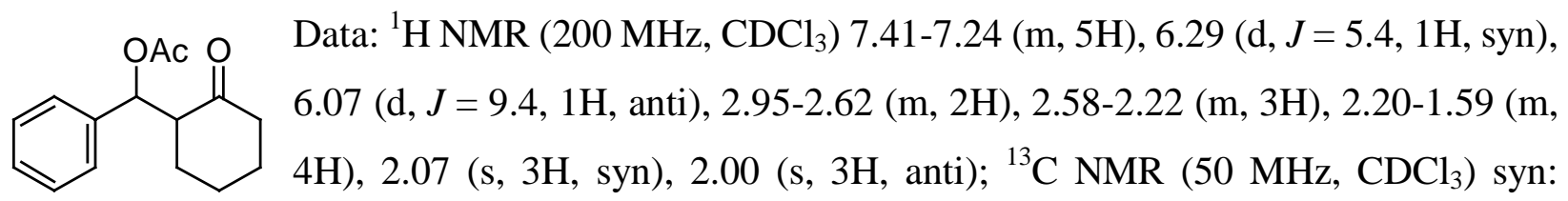
209.15, 169.63, 139.57, 128.16, 127.49, 126.26, 72.45, 55.53, 42.04, 27.57, 27.18, 24.35, 20.74. anti: $210.15,169.63,138.19,128.31,127.33,126.26,73.98,54.94,41.80,30.19,27.95,23.96$, 23.09; IR ( $\left.\mathrm{CH}_{2} \mathrm{Cl}_{2}\right) 2987$ (s), 1771 (m), 1742 (m), 1422 (s), 1250 (s), 1159 (m), 896 (s); MS (70 eV) 246 (M+ 84), 219 (19), 218 (35), 212 (33), 208 (100); TLC $\mathrm{R}_{f} 0.52$ (syn), 0.58 (anti) (EtOAc/hexane, 1/6).

\section{(2-Oxo-cyclohexyl)-phenyl-methyl 2,2-dimethylpropanoate}

Data: ${ }^{1} \mathrm{H}$ NMR (200 MHz, $\mathrm{CDCl}_{3}$ ) 7.51-7.23 (m, 5H), 6.26 (d, $J=4.6,1 \mathrm{H}$, syn), 6.03 (d, $J=9.2$, 1H, anti), 2.92-2.67 (m, 2H), 2.59-2.25 (m, 3H), 2.02-1.20 (m, 4H), 1.21 (s, 9H, syn), 1.14 (s, 9H, 


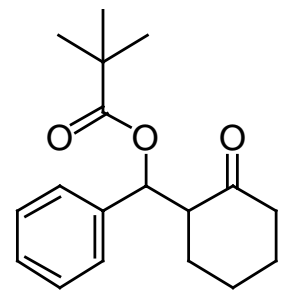

anti); ${ }^{13} \mathrm{C}$ NMR (50 MHz, $\mathrm{CDCl}_{3}$ ) syn: 208.97, 177.06, 139.89, 136.67, 128.51, $128.25,127.48,126.11,72.12,55.84,42.15,38.69,28.80,26.99,24.52,23.73$. anti: $209.95,177.06,138.61,135.56,128.39,128.31,128.03,127.19,73.92$, 55.35, 41.62, 38.42, 27.99, 26.83, 23.82, 23.23; IR $\left(\mathrm{CH}_{2} \mathrm{Cl}_{2}\right) 3032(\mathrm{w}), 2945$ (m), 2863 (m), 1725(m), 1698 (s), 1595 (m), 1522 (m), 1451 (m), 1347 (s), 1266 (m), 1131 (m), 853 (w); MS (70 eV) 288 (M $\left.\mathrm{M}^{+}, 9\right), 271$ (20), 270 (100); TLC R 0.67 (syn), 0.60 (anti) (EtOAc/hexane, 1/6).

\section{Dicetone-D-glucose-3-acetate ${ }^{57}$ and 6-acetate ${ }^{58}$}

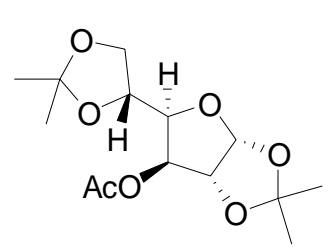

In a dry 25-mL, two-necked, round-bottomed flask was placed $\mathrm{MoO}_{2} \mathrm{Cl}_{2}(1.9$ $\mathrm{mg}, 0.01 \mathrm{mmol}$ ) in $5 \mathrm{~mL}$ of anhydrous $\mathrm{CH}_{2} \mathrm{Cl}_{2}$. To the above solution, acetic anhydride $(140 \mu \mathrm{L}, 1.5 \mathrm{mmol})$ was added at ambient temperature and stirred for one hour. A solution of diacetone-D-glucose $(260 \mathrm{mg}, 1.0 \mathrm{mmol})$ in $\mathrm{CH}_{2} \mathrm{Cl}_{2}(5 \mathrm{~mL})$ was slowly added to the above bluish solution. The reaction mixture was stirred for 60 hours at ambient temperature. After completion of the reaction as monitored by TLC, the reaction mixture was quenched with cold, saturated aqueous $\mathrm{NaHCO}_{3}$ solution $(20 \mathrm{~mL})$. The separated organic layer was washed with brine, dried $\left(\mathrm{MgSO}_{4}\right)$, filtered, and evaporated. The crude product was purified by column chromatography on silica gel (EtOAc/n-hexane, 1/5) to give the 3- and 6-acetate in $71 \%$ (214.2 mg) and $27 \%(82.5 \mathrm{mg})$ yields, respectively: ${ }^{1} \mathrm{H}$ NMR $\left(200 \mathrm{MHz}, \mathrm{CDCl}_{3}\right) 5.84(\mathrm{~d}, J=3.8,1 \mathrm{H}), 5.21(\mathrm{~d}, J=2.2,1 \mathrm{H}), 4.46(\mathrm{~d}, J=3.6,1 \mathrm{H}), 4.20-4.13(\mathrm{~m}$, $2 \mathrm{H}), 4.08-3.94(\mathrm{~m}, 2 \mathrm{H}), 2.06$ (s, 3H), $1.48(\mathrm{~s}, 3 \mathrm{H}), 1.37$ (s, 3H), $1.28(\mathrm{~s}, 3 \mathrm{H}), 1.27(\mathrm{~s}, 3 \mathrm{H}) ;{ }^{13} \mathrm{C}$ NMR (50 MHz, $\mathrm{CDCl}_{3}$ ) 169.68, 112.29, 109.35, 105.04, 83.33, 79.67, 76.11, 72.40, 67.14, 26.75, 26.63, 26.11, 25.17, 20.77; MS (70 eV) $303\left(\mathrm{M}+1^{+}, 100\right), 298$ (42), 293 (10); TLC $\mathrm{R}_{f} 0.26$ (EtOAc/hexane,1/5).

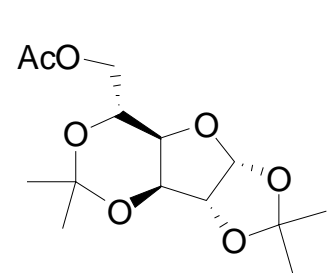

Data: ${ }^{1} \mathrm{H}$ NMR (200 MHz, $\left.\mathrm{CDCl}_{3}\right) 5.98(\mathrm{~d}, J=3.6,1 \mathrm{H}), 4.56(\mathrm{~d}, J=3.8,1 \mathrm{H})$, 4.34-4.09 (m, 5H), 3.80-3.71 (m, 1H), 2.07 (s, 3H), $1.48(\mathrm{~s}, 3 \mathrm{H}), 1.36(\mathrm{~s}, 3 \mathrm{H})$, 1.35 (s, 3H), 1.32 (s, 3H); ${ }^{13} \mathrm{C}$ NMR (50 MHz, $\mathrm{CDCl}_{3}$ ) 170.92, 112.29, 106.45, 101.09, 83.92, 79.41, 74.98, 70.14, 64.45, 27.07, 26.43, 23.82, 20.76; MS (70 eV) 303 (M+1 $\left.{ }^{+}, 100\right), 298$ (16), 289 (20); TLC R $\mathrm{R}_{f} 0.36$ (EtOAc/hexane, 1/5).

$\mathbf{O}^{2^{2}}, \mathbf{O}^{3^{\prime}}, \mathbf{O}^{5^{\prime}}$-Triacetyl-uridine $\mathbf{s}^{59}$ 


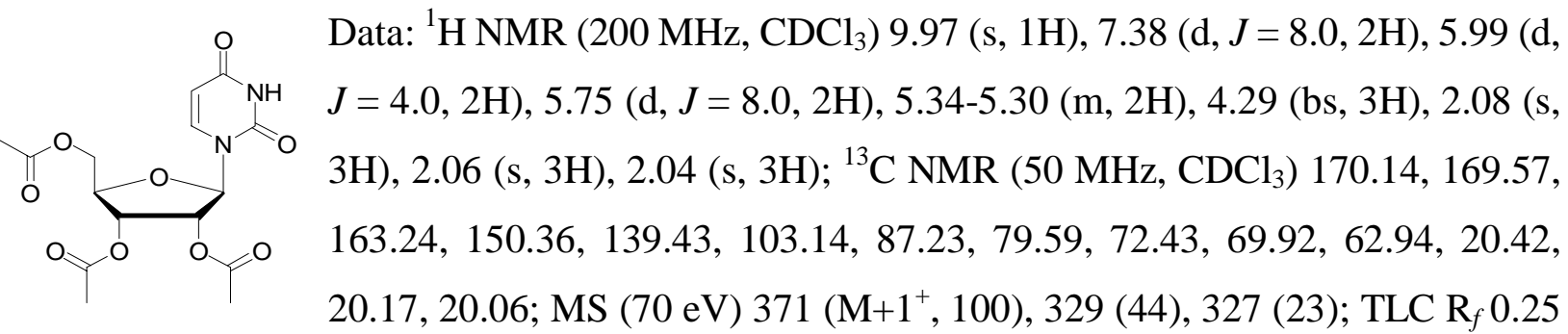

(EtOAc/hexane, 2/1).

\section{Acetic acid 4, 5, 6-triacetoxy-2-acetoxymethyl-tetrahydro-pyran-3-yl ester ${ }^{60}$}

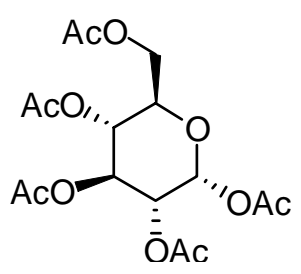

Data: ${ }^{1} \mathrm{H}$ NMR (200 MHz, $\left.\mathrm{CDCl}_{3}\right) 6.30(\mathrm{~d}, J=3.8,1 \mathrm{H}, \alpha$-form), 5.68 (dd, $J=$ 8.0, 1.6, $1 \mathrm{H}, \beta$-form), 5.45 (t, $J=8.2,1 \mathrm{H}, \beta$-form), 5.27-5.03 (m, $2 \mathrm{H})$, $4.32-4.20(\mathrm{~m}, 1 \mathrm{H}), 4.17-4.04(\mathrm{~m}, 1 \mathrm{H}), 3.84-3.77(\mathrm{~m}, 1 \mathrm{H}), 2.16,2.15,2.09,2.07$,

2.05, 2.00, 1.99, 1.98 (s, $15 \mathrm{H}, \alpha+\beta$ forms); ${ }^{13} \mathrm{C} \mathrm{NMR}\left(50 \mathrm{MHz}, \mathrm{CDCl}_{3}\right): 170.70$, 170.19, 169.34, 169.05, 168.84, 91.70, 89.06, 72.77, 72.71, 69.81, 67.87, 67.73, 61.42, 20.79, 20.71, 20.61, 20.47, 20.35; TLC $\mathrm{R}_{f} 0.35$ (ether/hexane, 1/2)

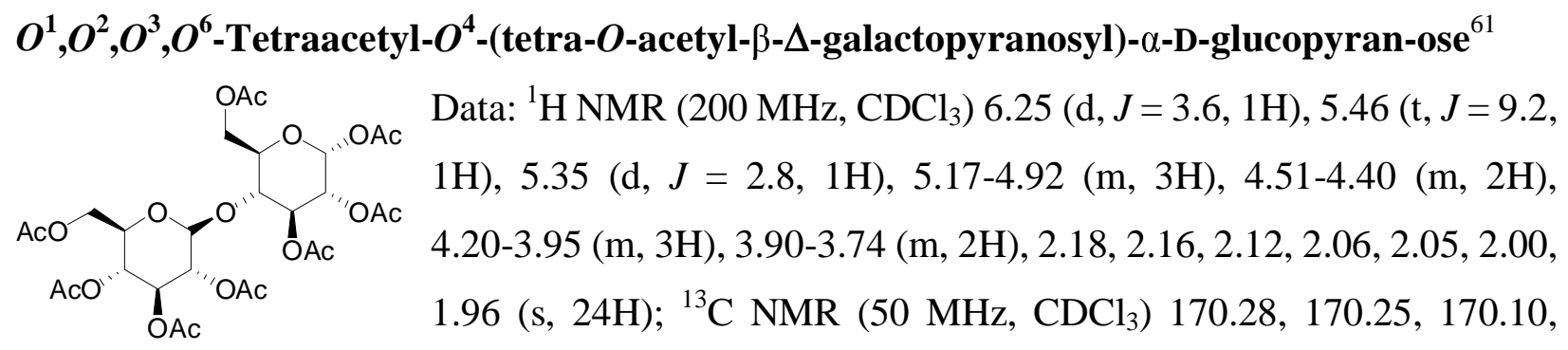
170.01, 169.88, 169.57, 169.08, 168.88, 101.05, 88.82, 75.64, 70.84, 70.55, 69.44, 69.26, 68.97, 66.47, 61.31, 60.66, 20.71, 20.62, 20.42, 20.27; MS (70 eV) $678\left(\mathrm{M}^{+}, 10\right), 677$ (25), $662(54), 648$ (33), 647 (79), 636 (27), 635 (100); TLC $\mathrm{R}_{f} 0.26$ (EtOAc/hexane, 1/1).

\section{Heneicosa- $O$-acetyl-cyclo-lin-hepta[1 $\alpha=>4]$-D-glucopyranosy ${ }^{62}$}

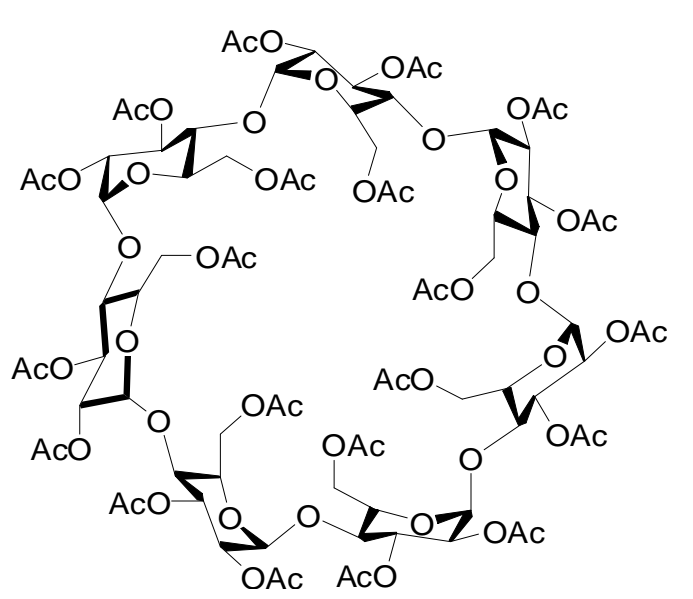

Data: ${ }^{1} \mathrm{H}$ NMR (400 MHz, $\left.\mathrm{CDCl}_{3}\right) 5.29(\mathrm{t}, J=9.4,7 \mathrm{H})$, $5.08(\mathrm{~d}, J=3.5,7 \mathrm{H}), 4.79(\mathrm{dd}, J=9.6,4.0,7 \mathrm{H}), 4.56(\mathrm{~d}$, $J=12.4,7 \mathrm{H}), 4.26(\mathrm{dd}, J=12.5,4.0,7 \mathrm{H}), 4.16-4.13(\mathrm{~m}$, $7 \mathrm{H}), 3.70(\mathrm{t}, J=8.2,7 \mathrm{H}), 2.12(\mathrm{~s}, 21 \mathrm{H}), 2.09(\mathrm{~s}, 21 \mathrm{H})$, $2.05(\mathrm{~s}, 21 \mathrm{H}) ;{ }^{13} \mathrm{C} \mathrm{NMR}\left(100 \mathrm{MHz}, \mathrm{CDCl}_{3}\right) 170.75$, 170.45, 169.46, 96.77, 76.76, 70.84, 70.40, 69.58, 62.45, 
20.62; IR (KBr) 2940 (m), 2340 (w), 1740 (s, C=O), 1370 (m), 1230 (s), 1040 (s), 900 (m); MS (FAB) $2017\left(\mathrm{M}^{+}, 12\right), 517$ (10), 169 (100); TLC R 0.32 (EtOAc/hexane, 4/1).

\section{Cellulose Tricetate ${ }^{63}$}

In a dry $50-\mathrm{mL}$, round-bottomed flask was placed cellulose $(500 \mathrm{mg})$ in a 1:1 solution of acetic acid and acetic anhydride $(10 \mathrm{~mL})$. The mixture was heated at $140{ }^{\circ} \mathrm{C}$ for 8 hours, then gradually cooled down to room temperature. A solution of $\mathrm{MoO}_{2} \mathrm{Cl}_{2}(10 \mathrm{mg})$ in acetic anhydride $(1 \mathrm{~mL})$ was slowly added at ambient temperature to above cellulose solution. The resultant mixture was heated at $100-110{ }^{\circ} \mathrm{C}$ for 6 hours. The reaction mixture was quenched with cold, saturated aqueous $\mathrm{NaHCO}_{3}$ solution $(50 \mathrm{~mL})$ and extracted with $\mathrm{CH}_{2} \mathrm{Cl}_{2}(3 \times 50 \mathrm{~mL})$. The combined organic layer was washed with brine, dried $\left(\mathrm{MgSO}_{4}\right)$, filtered, and evaporated. The crude white product was re-crystallized from $\mathrm{CH}_{2} \mathrm{Cl}_{2} /$ ether to give $850 \mathrm{mg}$ (96\% yield) of cellulose triacetate as white powder.

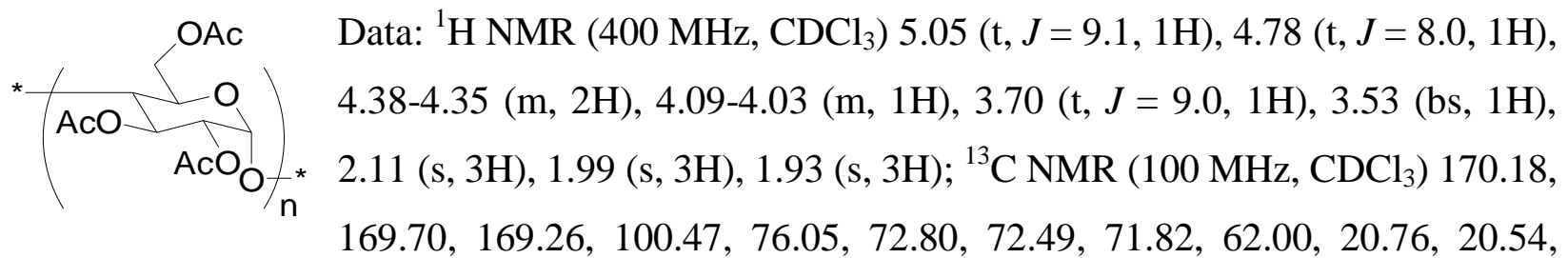

20.44; IR ( $\left.\mathrm{CH}_{2} \mathrm{Cl}_{2}\right) 3454$ (m), 2959 (m), 2893 (m), 2118 (w), 1751 (s), 1369 (s), 1232 (s), 1159 (s), 1123 (s), 1053 (s), $898(\mathrm{~m})$; TLC $\mathrm{R}_{f} 0.52\left(\mathrm{MeOH} / \mathrm{CH}_{2} \mathrm{Cl}_{2}, 1 / 9\right)$; GPC t $7.11 \min \left(\mathrm{M}_{\mathrm{n}} 8697, \mathrm{M}_{\mathrm{w}}\right.$ $\left.28811, \mathrm{M}_{\mathrm{w}} / \mathrm{M}_{\mathrm{n}}=3.31\right)$.

\section{(3-Hydroxy-[2]naphthyl)-methyl Acetate ${ }^{64}$}

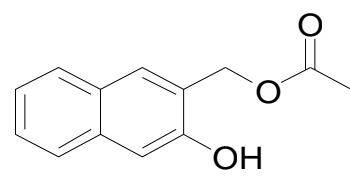

Data: ${ }^{1} \mathrm{H}$ NMR $\left(200 \mathrm{MHz}, \mathrm{CDCl}_{3}\right) 7.78$ (s, $\left.1 \mathrm{H}\right), 7.76-7.64(\mathrm{~m}, 2 \mathrm{H})$, 7.45-7.23 (m, 2H), 7.26 (s, 1H), 5.29 (s, 2H), 3.40 (bs, OH), 2.10 (s, 3H);

${ }^{13} \mathrm{C}$ NMR $\left(50 \mathrm{MHz}, \mathrm{CDCl}_{3}\right) 172.98,152.62,135.12,131.21,128.48$, 127.77, 126.85, 126.14, 124.15, 123.77, 111.38, 63.04, 20.80; IR $\left(\mathrm{CH}_{2} \mathrm{Cl}_{2}\right) 3050$ (s), 2987 (s), 1715 (m), 1640 (m), 1509 (m), 1422 (s), 1278 (s), 1106 (m), 1025 (m), 953 (m), 896 (s); MS (70 eV) $216\left(\mathrm{M}^{+}, 38\right), 156$ (38), 128 (100); TLC R 0.30 (EtOAc/hexane, 1/3).

\section{(3-Hydroxy-[2]naphthyl)-methyl 2,2-Dimethyl-propanoate}

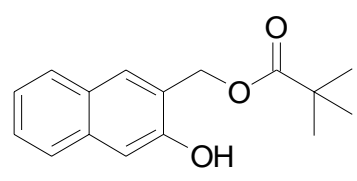

Data: ${ }^{1} \mathrm{H}$ NMR $\left(200 \mathrm{MHz}, \mathrm{CDCl}_{3}\right) 7.81(\mathrm{~s}, 1 \mathrm{H}), 7.74-7.65$ (m, 2H), 
7.43-7.26 (m, 2H), $7.26(\mathrm{~s}, 1 \mathrm{H}), 5.30(\mathrm{~s}, 2 \mathrm{H}), 1.20(\mathrm{~s}, 9 \mathrm{H}) ;{ }^{13} \mathrm{C} \mathrm{NMR}\left(50 \mathrm{MHz}, \mathrm{CDCl}_{3}\right)$ 180.62, 152.82, 135.22, 131.24, 128.51, 127.78, 126.84, 126.23, 124.46, 123.75, 111.70, 63.21, 38.87, 27.00; IR ( $\left.\mathrm{CH}_{2} \mathrm{Cl}_{2}\right) 3062$ (s), 2988 (s), 2686 (m), 2410 (m), 2306 (s), 1668 (w), 1554 (m), 1422 (s), 1281 (s), 1159 (m), 895 (s); MS (70 eV) 258 (M+2 25), 156 (100), 149 (10), 128 (82), 105 (39), 57 (40); TLC $\mathrm{R}_{f} 0.32$ (EtOAc/hexane, 1/3).

\section{(3-Hydroxy-[2]naphthyl)-methyl benzoate}

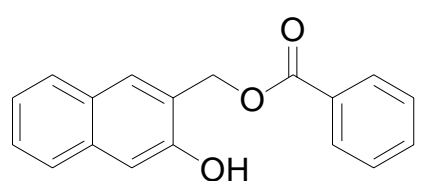

Data: ${ }^{1} \mathrm{H}$ NMR (400 MHz, $\left.\mathrm{CDCl}_{3}\right) 8.09(\mathrm{~d}, J=8.0,2 \mathrm{H}), 7.91(\mathrm{~s}, 1 \mathrm{H})$, $7.78(\mathrm{~d}, J=8.0,1 \mathrm{H}), 7.68(\mathrm{dd}, J=10.0,8.4,2 \mathrm{H}), 7.58(\mathrm{dd}, J=8.0,7.2$, 2H), 7.46-7.41 (m, 3H), 7.35-7.31 (m, 2H), $5.57(\mathrm{~s}, 2 \mathrm{H}),{ }^{13} \mathrm{C}$ NMR $(100$ $\left.\mathrm{MHz}, \mathrm{CDCl}_{3}\right)$ 168.36, 152.76, 135.35, 133.57, 131.87, 129.94, 129.32, 128.54, 128.46, 127.78, 126.96, 126.26, 124.13, 123.84, 112.06, 63.74; IR $\left(\mathrm{CH}_{2} \mathrm{Cl}_{2}\right) 3299$ (w), 3020 (w), 2361 (w), 2254 (m), $1724(\mathrm{w}), 1687$ (w), 1604 (w), 1380 (w), 1140 (w), $890(\mathrm{~s})$; MS (70 eV) $278\left(\mathrm{M}^{+}, 58\right), 156$ (85), 128 (100), 105 (38), 77 (22); TLC R 0.35 (EtOAc/hexane, 1/3).

\section{$N$-(1-Hydroxymethyl-2,2-dimethyl-propyl)-acetamide ${ }^{654}$}

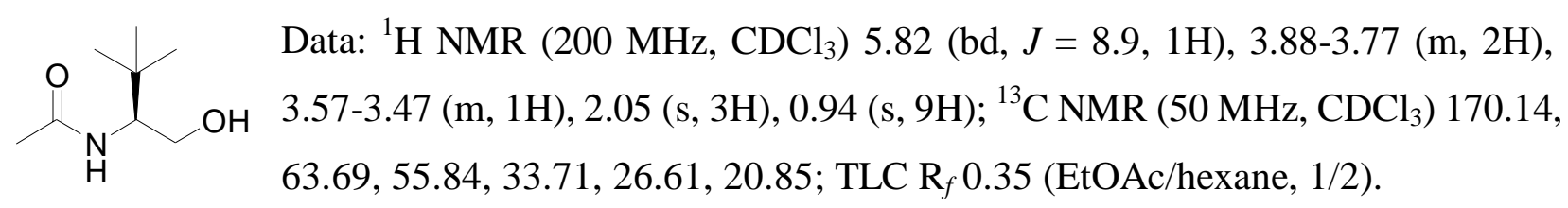

\section{$N$-(1-Hydroxymethyl-2,2-dimethyl-propyl)-2,2-dimethyl-propanamide ${ }^{66}$}

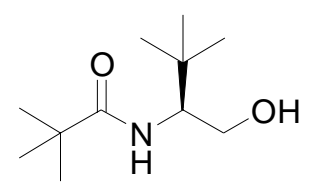

Data: ${ }^{1} \mathrm{H}$ NMR (200 MHz, $\left.\mathrm{CDCl}_{3}\right) 5.84$ (bs, 1H), 3.90-3.75 (m, 2H), 3.59-3.50 $(\mathrm{m}, 1 \mathrm{H}), 2.63$ (bs, 1H), 1.24 (s, 9H), $0.96(\mathrm{~s}, 9 \mathrm{H}) ;{ }^{13} \mathrm{C} \mathrm{NMR}\left(50 \mathrm{MHz}, \mathrm{CDCl}_{3}\right)$ 180.11, 63.71, 59.52, 38.98, 33.43, 27.57, 26.87; $\mathrm{R}_{f} 0.20$ (EtOAc/hexane, 1/5).

\section{$N$-(1-Hydroxymethyl-2,2-dimethyl-propyl)-benzamide ${ }^{67}$}

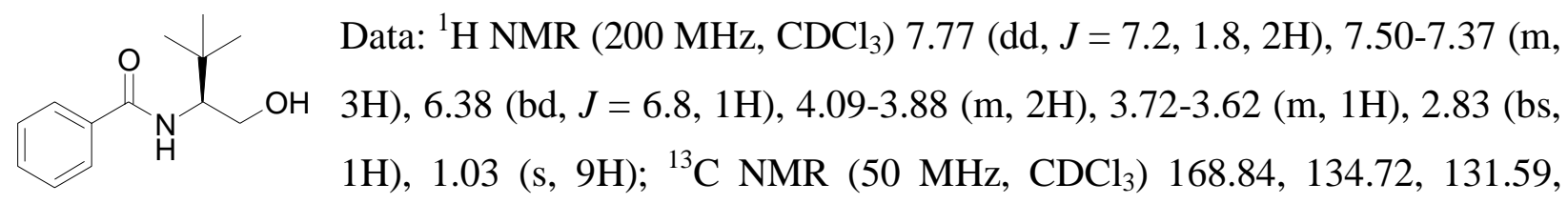
128.65, 126.99, 63.06, 59.81, 33.87, 30.79, 26.98; $\mathrm{R}_{f} 0.18$ (EtOAc/hexane, 1/1). 
(1-Hydroxymethyl-2,2-dimethyl-propyl)-carbamic acid tert-butyl ester ${ }^{68}$

$>_{\mathrm{O}}^{\mathrm{O}} \mathrm{H}_{\mathrm{H}}^{\mathrm{O}}$

Data: ${ }^{1} \mathrm{H}$ NMR (400 MHz, $\left.\mathrm{CDCl}_{3}\right) 4.61(\mathrm{bs}, 1 \mathrm{H}), 3.85$ (t, $\left.J=7.0,1 \mathrm{H}\right), 3.49$ (d, $\left(\mathrm{M}+1^{+}, 5\right), 186$ (15), 162 (35), 144 (15), 130 (39), 104 (13), 86 (45), 60 (37), 57 (100); $\mathrm{R}_{f} 0.26$ (EtOAc/hexane, 1/5).

N-(2-Hydroxy-1,1-dimethyl-ethyl)-acetamide ${ }^{69}$

Data: ${ }^{1} \mathrm{H}$ NMR $\left(400 \mathrm{MHz} \mathrm{CDCl}_{3}\right) 6.26(\mathrm{~s}, 1 \mathrm{H}), 5.28(\mathrm{~s}, 1 \mathrm{H}), 3.49(\mathrm{~s}, 2 \mathrm{H}), 1.92$
$(\mathrm{~s}, 3 \mathrm{H}), 1.23(\mathrm{~s}, 6 \mathrm{H}) ;{ }^{13} \mathrm{C} \mathrm{NMR}\left(100 \mathrm{MHz}, \mathrm{CDCl}_{3}\right)$ 171.45, 70.50, 55.87, 24.29, 23.74; MS (20 eV) $132\left(\mathrm{M}+1^{+}, 4\right), 128$ (16), 118 (24), 98 (20), 91 (44), 72 (38), 57 (100); TLC R 0.4 (EtOAc/hexane, 1/4).

\section{N-(2-Hydroxy-1,1-dimethyl-ethyl)-2, 2-dimethyl-propionamide}

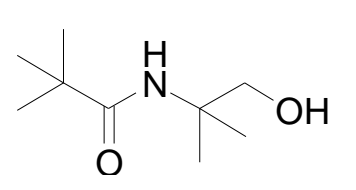

Data: ${ }^{1} \mathrm{H}$ NMR (400 MHz, $\left.\mathrm{CDCl}_{3}\right) 5.62$ (s, 1H), 4.98 (s, 1H), 3.57 (s, 2H), $1.28(\mathrm{~s}, 6 \mathrm{H}), 1.19(\mathrm{~s}, 9 \mathrm{H}) ;{ }^{13} \mathrm{C} \mathrm{NMR}\left(100 \mathrm{MHz}, \mathrm{CDCl}_{3}\right)$ 179.54, 70.74, 55.47, 38.86, 27.50, 24.39; IR $\left(\mathrm{CH}_{2} \mathrm{Cl}_{2}\right) 3449$ (m), 3314 (m), 2967 (s), 2878 (m),

1643 (s), 1472 (m), 1368 (m), 1210 (m), 1073 (m); MS (20 eV) 174 (M+1 + 100), 142 (32), 102 (33), 69 (26), 57 (98); TLC R 0.36 (EtOAc/hexane, 1/4).

\section{N-(2-Hydroxy-1,1-dimethyl-ethyl)-Benzoate ${ }^{70}$}

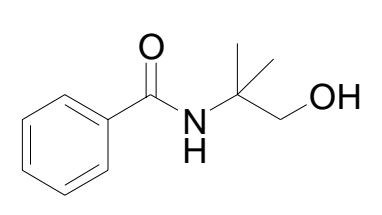

Data: ${ }^{1} \mathrm{H}$ NMR (400 MHz, $\left.\mathrm{CDCl}_{3}\right)$ 7.71-7.23 (m, 2H), 7.49-7.38 (m, 3H), $6.31(\mathrm{bs}, 1 \mathrm{H}), 5.08$ (bs, 1H), $3.68(\mathrm{~s}, 2 \mathrm{H}), 1.41$ (s, 6H); ${ }^{13} \mathrm{C} \mathrm{NMR}(50 \mathrm{MHz}$, $\left.\mathrm{CDCl}_{3}\right)$ 168.41, 134.80, 131.54, 128.53, 126.86, 70.56, 56.34, 24.66, 24.56; MS (20 eV) $194\left(\mathrm{M}+1^{+}, 15\right), 162$ (51), 122 (14), 105 (100), 77 (28); R 0.38 (EtOAc/hexane, 1/4).

(2-Hydroxy-1,1-dimethyl-ethyl)-carbamic acid tert-butyl ester ${ }^{71}$

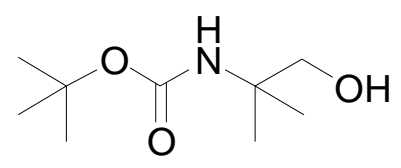

Data: ${ }^{1} \mathrm{H}$ NMR (400 MHz, $\left.\mathrm{CDCl}_{3}\right) 4.76(\mathrm{~s}, 1 \mathrm{H}), 3.53(\mathrm{~s}, 2 \mathrm{H}), 1.42$ (s, 6H), $1.24(\mathrm{~s}, 9 \mathrm{H}) ;{ }^{13} \mathrm{C} \mathrm{NMR}\left(100 \mathrm{MHz}, \mathrm{CDCl}_{3}\right)$ 156.08, 79.61, 70.66, 54.15, 28.31, 24.53; MS (20 eV) $190\left(\mathrm{M}+1^{+}, 39\right), 158$ (13), 134 (36), 116 (16), 102 (17), 86 (42), 84 (100), 58 (44); TLC R 0.33 (EtOAc/hexane, 1/9). 
cis-octadec-9-enoic acid 1-phenethyl-but-3-enyl ester (1a)

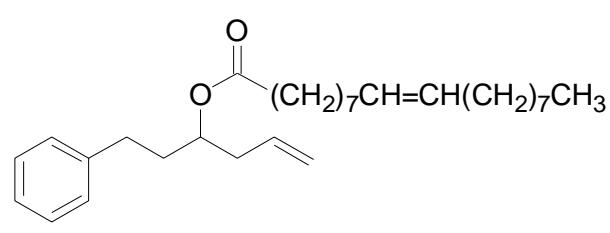

In a dry 50-mL, two-necked, round-bottomed flask was charged with $\mathrm{MoO}_{2} \mathrm{Cl}_{2}(9.5 \mathrm{mg}, 0.05 \mathrm{mmol})$ in anhydrous $\mathrm{CH}_{2} \mathrm{Cl}_{2}(2 \mathrm{~mL})$. To the above solution of catalyst, oleic acid (297 $\mathrm{mg}, 1.05 \mathrm{mmol}$ in $2 \mathrm{~mL}$ of $\mathrm{CH}_{2} \mathrm{Cl}_{2}$ ) was added at ambient temperature followed by addition of benzoic anhydride $(249 \mathrm{mg}, 1.1 \mathrm{mmol}$ in $2 \mathrm{~mL}$ of $\mathrm{CH}_{2} \mathrm{Cl}_{2}$ ). A solution of 1-phenethyl-but-3-en-1-ol (176.2 $\left.\mathrm{mg}, 1.0 \mathrm{mmol}\right)$ in $\mathrm{CH}_{2} \mathrm{Cl}_{2}(2 \mathrm{~mL})$ was added after $15 \mathrm{~min}$. The resultant reaction mixture was stirred for $6 \mathrm{~h}$ and then quenched with cold, saturated aqueous $\mathrm{NaHCO}_{3}$ solution $(10 \mathrm{~mL})$. The separated organic layer was washed with brine, dried over $\mathrm{MgSO}_{4}$, filtered, and evaporated. The resultant crude product was purified by column chromatography (EtOAc/hexane, 3/97) on silica gel to furnish $338 \mathrm{mg}$ (95\% yield) of the pure oleate: ${ }^{1} \mathrm{H}$ NMR (200 MHz, $\left.\mathrm{CDCl}_{3}\right)$ 7.32-7.14 (m, 5H), 5.82-5.68 (m, 1H), $5.34(\mathrm{t}, J=5.4,2 \mathrm{H})$, $5.12-4.95(\mathrm{~m}, 3 \mathrm{H}), 2.63(\mathrm{q}, J=7.0,2 \mathrm{H}), 2.35(\mathrm{t}, J=6.8,2 \mathrm{H}), 2.28(\mathrm{t}, J=7.6,2 \mathrm{H}), 2.02-1.81(\mathrm{~m}$, $3 \mathrm{H}), 1.65-1.62(\mathrm{~m}, 2 \mathrm{H}), 1.38-1.22(\mathrm{~m}, 21 \mathrm{H}), 0.88(\mathrm{t}, J=6.5,3 \mathrm{H}) ;{ }^{13} \mathrm{C} \mathrm{NMR}\left(50 \mathrm{MHz}, \mathrm{CDCl}_{3}\right)$ $173.30,141.45,133.50,129.89,129.65,128.32,128.27,125.84,117.65,72.31,38.60,35.22$, $34.37,31.76,31.62,29.60,29.54,29.38,29.16,29.03,28.98,28.95,27.05,26.99,24.90,22.52$, 13.93; IR ( $\left.\mathrm{CH}_{2} \mathrm{Cl}_{2}\right) 3029$ (m), 2937 (s), 2856 (s), 1713 (s), 1606 (m), 1447 (m), 1316 (m), 1266 (w), 1174 (m), 1116 (m), 867 (w); MS (70 eV) 356 (M+, 5), 355 (16), 341 (17), 298 (32), 296 (100), 292 (56), 281 (32), 280 (48), 177 (18), 158 (20), 149 (50), 117 (100), 105 (64), 91 (36); R 0.5 (EtOAc/hexane, 3/97).

\section{2-(9H-Fluoren-9-yl-methoxycarbonylamino)-4-methyl-pentanoic acid 1-phenethyl-} but-3-enyl ester (1b)

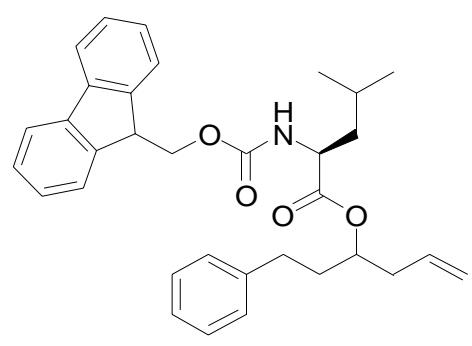

Data: ${ }^{1} \mathrm{H}$ NMR (400 MHz, $\left.\mathrm{CDCl}_{3}\right) 7.80(\mathrm{~d}, J=7.6,2 \mathrm{H}), 7.67-7.62$ (m, 2H), 7.45-7.18 (m, 9H), 5.81-5.74 (m, 1H), 5.29-5.04 (m, 4H), 4.47-4.42 (m, 3H), $4.27(\mathrm{t}, J=6.8,1 \mathrm{H}), 2.75-2.61(\mathrm{~m}, 2 \mathrm{H}), 2.42(\mathrm{t}$, $J=6.8,2 \mathrm{H}), 2.02-1.91(\mathrm{~m}, 2 \mathrm{H}), 1.79-1.51(\mathrm{~m}, 3 \mathrm{H}), 1.04-1.00(\mathrm{~m}$, $6 \mathrm{H}) ;{ }^{13} \mathrm{C} \mathrm{NMR}\left(100 \mathrm{MHz}, \mathrm{CDCl}_{3}\right)$ 172.69, 155.86, 143.87, 143.70, 141.23, 133.06, 132.96, 128.32, 127.60, 126.96, 125.90, 124.98, 119.88, 118.09, 73.97, 66.82, 52.62, 47.14, 41.80, 38.46, 35.11, 31.52, 24.72, 22.84, 21.77; IR $\left(\mathrm{CH}_{2} \mathrm{Cl}_{2}\right) 3945$ (m), 3056 (s), 2960 (s), 1726 (s), 1648 (m), 1422 (s), 1277 (s), 1252 (s), 896 (s); MS (ESI) 543 (M+MeOH, 30), 
$542\left(\mathrm{M}^{+}+\mathrm{OMe}, 37\right), 399$ (17), 181 (100); TLC R 0.21 (EtOAc/hexane, 1/9).

2-Oxo-propionic acid 1-phenethyl-but-3-enyl ester (1c)

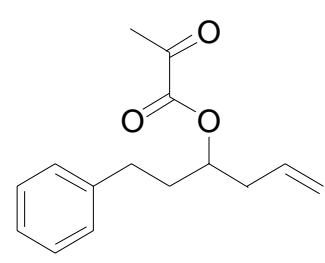

Data: ${ }^{1} \mathrm{H}$ NMR (400 MHz, $\left.\mathrm{CDCl}_{3}\right)$ 7.30-7.15 (m, 5H), 5.79-5.69 (m, 1H), 5.13-5.05 (m, 3H), 2.72-2.60 (m, 2H), 2.45-2.41 (m, 2H), $2.44(\mathrm{~s}, 3 \mathrm{H})$, 2.12-1.95 (m, 2H); ${ }^{13} \mathrm{C}$ NMR (100 MHz, $\left.\mathrm{CDCl}_{3}\right)$ 191.97, 160.54, 140.92, 132.72, 128.50, 128.30, 126.13, 118.57, 75.77, 38.50, 34.97, 31.67, 26.74; IR $\left(\mathrm{CH}_{2} \mathrm{Cl}_{2}\right) 3491$ (m), 3052 (s), 1738 (s), 1694 (s), 1605 (m), 1585 (m), 1420 (s), 1319 (s), 1287 (s), 1250 (s), 1177 (m), 1071 (m), 1026 (m), 896 (s); MS (70 eV) 246 (M+2 2), 205 (13), 158 (24), 133 (13), 117 (100), 104 (21), 91 (69); TLC R 0.31 (EtOAc/hexane, 1/9).

2-(9H-Fluoren-9-yl-methoxycarbonylamino)-4-methyl-pentanoic acid benzhydryl ester (2a)

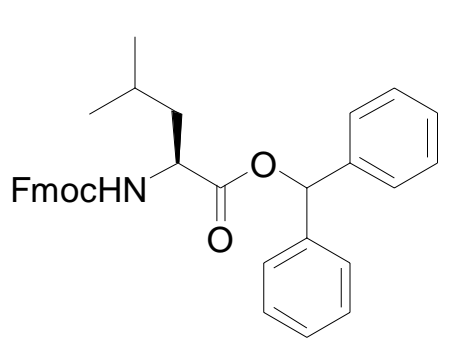
Data: ${ }^{1} \mathrm{H}$ NMR (400 MHz, $\left.\mathrm{CDCl}_{3}\right) 7.77(\mathrm{~d}, J=7.5,2 \mathrm{H}), 7.59$ (d, $J=$ $7.4,2 \mathrm{H}), 7.42-7.28(\mathrm{~m}, 14 \mathrm{H}), 6.91(\mathrm{~s}, 1 \mathrm{H}), 5.19(\mathrm{~d}, J=8.7,1 \mathrm{H})$, $4.57-4.52(\mathrm{~m}, 1 \mathrm{H}), 4.39(\mathrm{~d}, J=6.8,2 \mathrm{H}), 4.21(\mathrm{t}, J=7.0,1 \mathrm{H})$, 1.70-1.53 (m, 3H), 0.95-0.92 (m, 6H); ${ }^{13} \mathrm{C}$ NMR (100 MHz, $\left.\mathrm{CDCl}_{3}\right)$ $172.11,155.89,143.90,143.70,141.25,139.59,139.52,128.49$,

128.06, 127.99, 127.63, 127.14, 127.00, 126.94, 125.03, 119.91, 77.91, 66.95, 52.67, 47.15, 41.58, 24.68, 22.79, 21.83; IR ( $\left.\mathrm{CH}_{2} \mathrm{Cl}_{2}\right) 3439$ (m), 3057 (s), 2986 (s), 1726 (s), 1609 (m), 1511 (m), 1422 (s), $1384(\mathrm{~m}), 1250(\mathrm{~s}), 912(\mathrm{~s}) ; \mathrm{MS}(20 \mathrm{eV}) 519\left(\mathrm{M}^{+}, 5\right), 178$ (100), 165 (10), 91 (10) ; TLC R 0.28 (EtOAc/hexane, 1/9).

\section{2-Oxo-propionic acid benzhydryl ester (2b)}

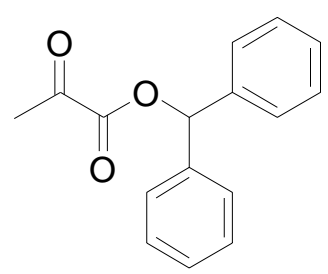

Data: ${ }^{1} \mathrm{H}$ NMR (400 MHz, $\left.\mathrm{CDCl}_{3}\right)$ 7.41-7.30 (m, 10H), 6.96 (s, 1H), 2.49 (s, $3 \mathrm{H}) ;{ }^{13} \mathrm{C}$ NMR (100 MHz, $\left.\mathrm{CDCl}_{3}\right)$ 191.43, 159.85, 138.93, 128.65, 128.35, 127.12, 78.98, 26.73; IR $\left(\mathrm{CH}_{2} \mathrm{Cl}_{2}\right) 3033$ (m), 2958 (m), 1735 (s), 1598 (m),

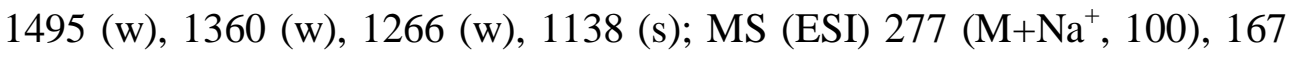
(33); TLC R 0.26 (EtOAc/hexane, 1/9).

\section{$N$-Fmoc-L-leucyl-L-tert-leucine methyl ester (3)}

In a dry $50-\mathrm{mL}$, two-necked, round-bottomed flask was charged with $\mathrm{MoO}_{2} \mathrm{Cl}_{2}(5.0 \mathrm{mg}$, $0.025 \mathrm{mmol}, 5 \mathrm{~mol} \%)$ in anhydrous $\mathrm{CH}_{2} \mathrm{Cl}_{2}(5 \mathrm{~mL})$. To the above solution of catalyst, 
Fmoc- $L$-leucine $\left(176 \mathrm{mg}, 0.5 \mathrm{mmol}\right.$ in $5 \mathrm{~mL}$ of $\mathrm{CH}_{2} \mathrm{Cl}_{2}$ ) was added at ambient temperature followed by addition of benzoic anhydride $\left(170 \mathrm{mg}, 0.75 \mathrm{mmol}\right.$ in $5 \mathrm{~mL}$ of $\mathrm{CH}_{2} \mathrm{Cl}_{2}$. A solution of methyl L-tert-leucinate $(80.2 \mathrm{mg}, 0.6 \mathrm{mmol})$ in $\mathrm{CH}_{2} \mathrm{Cl}_{2}(5 \mathrm{~mL})$ was added after $60 \mathrm{~min}$. The resultant reaction mixture was stirred for $8 \mathrm{~h}$ and then quenched with cold, saturated aqueous $\mathrm{NaHCO}_{3}$ solution $(20 \mathrm{~mL})$. The aqueous layer was separated and extracted with $\mathrm{CH}_{2} \mathrm{Cl}_{2}(2 \times 15$ $\mathrm{mL}$ ). The combined organic extracts were washed with brine, dried over $\mathrm{MgSO}_{4}$, filtered, and evaporated. The resultant crude product was purified by column chromatography (EtOAc/n-hexane, 1/4) on silica gel to furnish $216 \mathrm{mg}$ (90\% yield) of the pure di-peptide.

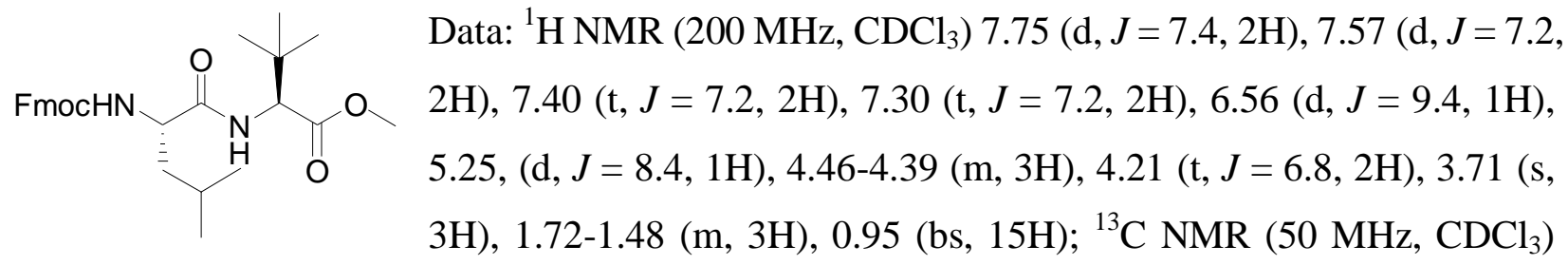

$171.89,171.75,156.34,143.88,143.77,141.36,127.77,127.13,125.07,120.01,67.11,60.04$, 53.56, 51.74, 47.10, 40.97, 34.75, 26.43, 24.58, 22.82, 21.94; IR ( $\left.\mathrm{CCl}_{4}\right) 3424$ (m), 3334 (m), 3062 (m), 3025 (m), 2959 (s), 2872 (m), 1946 (w), 1901 (w), 1740 (s), 1681 (s), 1512 (s), 1478 (s), 1450 (s), 1436 (s), 1401 (m), 1370 (m), 1340 (m), 1218 (s), 1166 (s), 1105 (m), 1044 (m), 995 (w), 936 (w), 856 (w); MS (70 eV) 480 (M+, 12), 465 (30), 449 (10), 424 (15), 386 (10), 308 (100), 285 (25), 269 (92), 264 (84), 243 (22), 237 (72); TLC R 0.3 (EtOAc/hexane, 1/4).

\section{2-(9H-Fluoren-9-yl-methoxycarbonylamino)-4-methyl-pentanethioic acid $S$-tert- butyl ester} (4)

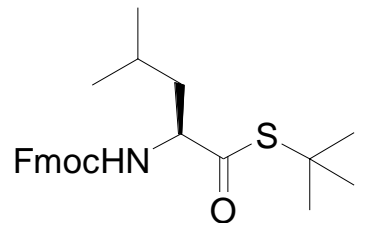

Data: ${ }^{1} \mathrm{H}$ NMR $\left(400 \mathrm{MHz}, \mathrm{CDCl}_{3}\right) 7.77$ (d, $\left.J=7.6,2 \mathrm{H}\right), 7.61(\mathrm{t}, J=7.6$, 2H), $7.40(\mathrm{t}, J=7.4,2 \mathrm{H}), 7.31(\mathrm{~d}, J=7.4,2 \mathrm{H}), 5.09(\mathrm{~d}, J=9.0,1 \mathrm{H})$, 4.82-4.34 (m, 3H), $4.25(\mathrm{t}, J=7.0,2 \mathrm{H}), 1.72-1.64(\mathrm{~m}, 3 \mathrm{H}), 1.47(\mathrm{~s}, 9 \mathrm{H})$, 0.96 (s, 3H), 0.95 (s, 3H); ${ }^{13} \mathrm{C}$ NMR (100 MHz, $\left.\mathrm{CDCl}_{3}\right)$ 201.46, 155.71, 143.95, 143.77, 141.34, 127.69, 127.05, 125.09, 119.97, 66.99, 59.69, 48.19, 47.25, 42.27, 29.78, 24.77, 23.10, 21.69; IR ( $\left.\mathrm{CH}_{2} \mathrm{Cl}_{2}\right) 3431$ (m), 3225 (m), 2989 (s), 2871 (s), 1955 (m), 1725 (s), 1681 (m), 1491 (s), 1448 (s), 1381 (s), 1351 (s), 1298 (s), 1119 (s), 1041 (s), 934 (s), 917 (m), 844 (s); MS (ESI) $425\left(\mathrm{M}^{+}, 100\right)$; TLC R 0.46 (EtOAc/hexane, 1/20).

\section{(1-tert-Butylcarbamoyl-3-methyl-butyl)-carbamic acid 9H-fluoren-9-ylmethyl ester (5) ${ }^{72}$}




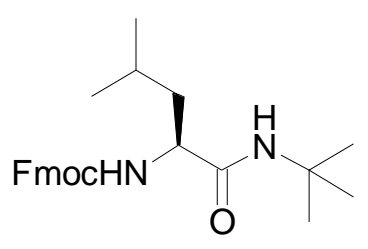

Data: ${ }^{1} \mathrm{H}$ NMR (400 MHz, $\left.\mathrm{CDCl}_{3}\right) 7.76(\mathrm{~d}, J=7.5,2 \mathrm{H}), 7.59(\mathrm{t}, J=7.5$, 2H), $7.40(\mathrm{t}, J=7.4,2 \mathrm{H}), 7.31(\mathrm{t}, J=7.4,2 \mathrm{H}), 5.71(\mathrm{bs}, 1 \mathrm{H}), 5.22(\mathrm{bd}, J=$ $6.9,1 \mathrm{H}), 4.41(\mathrm{~d}, J=6.8,2 \mathrm{H}), 4.22(\mathrm{t}, J=7.0,1 \mathrm{H}), 4.04-4.03(\mathrm{~m}, 1 \mathrm{H})$, 1.64-1.51 (m, 3H), 1.34 (s, 9H), 0.95 (s, 3H), 0.94 (s, 3H); ${ }^{13} \mathrm{C}$ NMR (100 $\mathrm{MHz}, \mathrm{CDCl}_{3}$ ) 171.27, 156.22, 143.81, 143.74, 141.29, 127.70, 127.05, 125.01, 119.96, 119.95, 66.93, 54.02, 51.38, 47.17, 41.77, 28.67, 24.70, 22.90, 22.14; IR $\left(\mathrm{CH}_{2} \mathrm{Cl}_{2}\right) 3425$ (m), 3052 (s), 2959 (m), 2748 (w), 2598 (w), 2483 (w), 2082 (w), 1718 (s), 1680 (s), 1560 (m), 1508 (s), 1422 (m), 1265 (s), 1216 (m), 1121 (m), 1050 (m), 896 (s); MS (ESI) $432\left(\mathrm{M}+\mathrm{Na}^{+}, 100\right), 410\left(\mathrm{M}^{+} \mathrm{H}^{+}\right.$, 35); TLC R 0.38 (EtOAc/hexane, 1/9).

\section{2-Methyl-acrylic acid trityl ester $(6)^{73}$}

In a dry $50 \mathrm{~mL}$, two-necked, round-bottomed flask was placed $\mathrm{MoO}_{2} \mathrm{Cl}_{2}(4 \mathrm{mg}, 0.02 \mathrm{mmol}$, $1 \mathrm{~mol} \%)$ in $5 \mathrm{~mL}$ of anhydrous toluene. A solution of methacrylic acid (205 $\mu \mathrm{L}, 2.4 \mathrm{mmol}, 1.2$ equiv) and benzoic anhydride (543 mg, $2.4 \mathrm{mmol}, 1.2$ equiv) in toluene (5 mL) was slowly added to the above solution. The resulting reaction mixture was stirred for 1 hour. To the above solution was slowly added a solution of diisopropylethylamine (350 $\mu \mathrm{L}, 2 \mathrm{mmol}, 1$ equiv) and trityl alcohol $(520 \mathrm{mg}, 2 \mathrm{mmol})$ in toluene $(5 \mathrm{~mL})$ at ambient temperature. This mixture was heated to reflux for 3 hours. After completion of the reaction as monitored by TLC, the reaction mixture was gradually warmed to room temperature and quenched with cold, saturated aqueous $\mathrm{NaHCO}_{3}$ solution $(20 \mathrm{~mL})$. The separated organic layer was washed with brine, dried $\left(\mathrm{MgSO}_{4}\right)$, filtered, and evaporated. The crude product was purified by column chromatography on silica gel to give $571 \mathrm{mg}(87 \%)$ of colorless oil along with $33 \mathrm{mg}(9 \%)$ benzoylation side product.

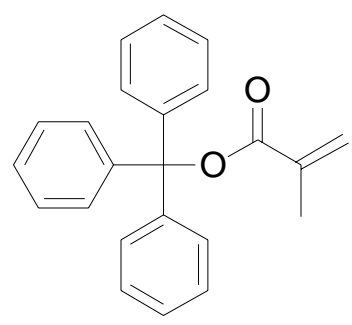

Data: ${ }^{1} \mathrm{H}$ NMR (400 MHz, $\left.\mathrm{CDCl}_{3}\right)$ 7.41-7.25 (m, 15H), 6.25-6.24 (m, 1H), $5.62-5.61(\mathrm{~m}, 1 \mathrm{H}), 2.00(\mathrm{dd}, J=1.24,1.20,3 \mathrm{H}) ;{ }^{13} \mathrm{C} \mathrm{NMR}(100 \mathrm{MHz}$, $\left.\mathrm{CDCl}_{3}\right)$ 165.23, 143.41, 137.49, 128.30, 127.91, 127.23, 125.58, 89.97, 18.56; TLC $\mathrm{R}_{f} 0.52$ (EtOAc/hexane/Et $\left.{ }_{3} \mathrm{~N}, 10 / 90 / 5\right)$.

\section{Molybdenum dichloride dipivalate oxide-I}

$\mathrm{Cl} \stackrel{\mathrm{MI} O}{\mathrm{Cl}}$

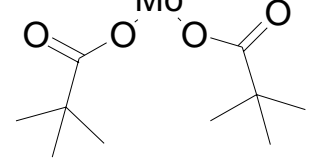

In a dry 50-mL, round-bottomed flask was placed $\mathrm{MoOCl}_{4}(127 \mathrm{mg}, 0.5 \mathrm{mmol})$ in $5 \mathrm{~mL}$ of anhydrous $\mathrm{CH}_{2} \mathrm{Cl}_{2}$. Silver pivalate $(209 \mathrm{mg}, 1.0 \mathrm{mmol})$ was added 
as a solid to the above solution and the reaction mixture was refluxed for 8 hours. This solution was allowed to cool down to the room temperature. White precipitate was observed and filtered. The filtrate was concentrated and dried in vacuo to give $\mathbf{I}$ as dark-green power $(173 \mathrm{mg}, 90 \%):{ }^{1} \mathrm{H}$ NMR (400 MHz, $\left.\mathrm{CDCl}_{3}\right) 1.251$ (s, $\left.\left.9 \mathrm{H}\right) ;{ }^{13} \mathrm{C} \mathrm{NMR} \mathrm{(100} \mathrm{MHz,} \mathrm{CDCl}_{3}\right)$ 185.87, 38.75, 26.89; MS (ESI) $450\left(\mathrm{M}+\mathrm{CH}_{3} \mathrm{CN}+\mathrm{Na}^{+}, 100\right), 410\left(\mathrm{M}+\mathrm{Na}^{+}, 6\right)$.

\section{Applications on Highly Functionalized Substrates:}

Acetic acid 2-(2-\{4-[2-(2-\{4-[2-(2-acetoxy-ethoxy)-ethoxy]-3,5-bis- allyloxy-benzoyl amino\}-ethyldisulfanyl)-ethylcarbamoyl]-2,6-bis-allyloxy-phenoxy\}-thoxy)-ethyl ester (7a)

In a dry $50 \mathrm{~mL}$, two-necked, round-bottomed flask was placed molybdenum dioxydichloride ( $2 \mathrm{mg}, 0.01 \mathrm{mmol}, 13 \mathrm{~mol} \%, 0.065$ equiv) in $15 \mathrm{~mL}$ of anhydrous $\mathrm{CH}_{2} \mathrm{Cl}_{2}$. To the above solution, acetic anhydride ( $35 \mu \mathrm{L}, 0.37 \mathrm{mmol}, 2.5$ equiv) was slowly added at ambient temperature. After for $10 \mathrm{~min}$, a solution of corresponding alcohol (60 mg, $0.076 \mathrm{mmol}$ in $\mathrm{CH}_{2} \mathrm{Cl}_{2}, 2 \mathrm{~mL}$ ) was slowly added to the above dark bluish green solution and the reaction mixture were stirred for 30 hours at room temperature. After completion, reaction mixture was quenched with cold, saturated aqueous $\mathrm{NH}_{4} \mathrm{Cl}$ solution $(5 \mathrm{~mL})$ and extracted with dichloromethane $(2 \times 5 \mathrm{~mL})$. The separated organic layer was washed with brine, dried $\left(\mathrm{MgSO}_{4}\right)$, filtered, and evaporated. The crude product was purified by column chromatography. The product obtained as colorless oil (63 mg, $95 \%)$ was characterized by routine spectroscopic methods.

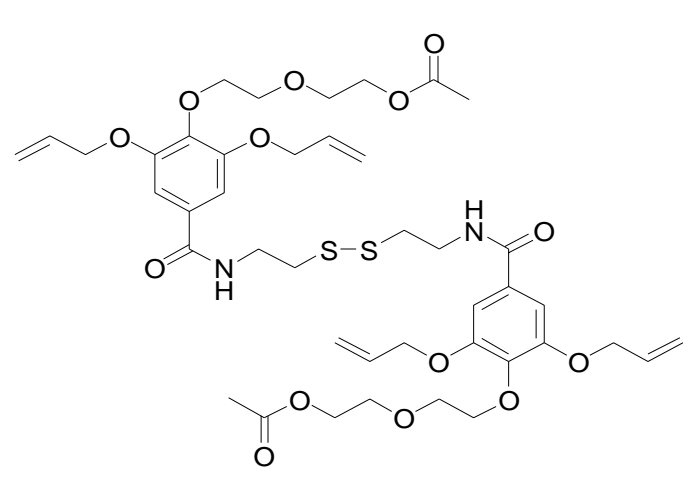

Data: ${ }^{1} \mathrm{H}$ NMR $\left(500 \mathrm{MHz}, \mathrm{CDCl}_{3}\right) 7.53(\mathrm{t}, J=5.6,1 \mathrm{H})$, $7.09(\mathrm{~s}, 2 \mathrm{H}), 5.95-5.87(\mathrm{~m}, 2 \mathrm{H}), 5.30(\mathrm{dd}, J=17.2,1.0$, 2H), $5.17(\mathrm{dd}, J=10.6,1.0,2 \mathrm{H}), 4.46(\mathrm{~d}, J=5.1,4 \mathrm{H})$, 4.16-4.13, (m, 4H), $3.76(\mathrm{t}, J=5.0,2 \mathrm{H}), 3.72(\mathrm{t}, J=4.9$, 2H), $3.69(\mathrm{t}, J=6.1,2 \mathrm{H}), 2.89(\mathrm{t}, J=6.4,2 \mathrm{H}), 2.01(\mathrm{~s}$, 3H). ${ }^{13} \mathrm{C}$ NMR (125 MHz, $\left.\mathrm{CDCl}_{3}\right)$ 170.94, 167.26, 152.17, 140.90, 132.77, 129.11, 117.48, 106.54, 72.26, 70.34, 69.78, 68.88, 63.60, 39.32, 37.76, 20.80; IR (CCl $) 3355$ (w), 2927 (m), 1743 (s), 1649 (m), 1581 (m), 1493 (s), 1421 (m), 1364 (w), 1330 (s), 1232 (s), 1132 (s), 929 (m); MS (70 eV) 439 (M/2+, 40), 423 (38), 362 (20), 232 (20), 215 (22), 87 (100); Anal. Calcd. for $\mathrm{C}_{42} \mathrm{H}_{56} \mathrm{~N}_{2} \mathrm{O}_{14} \mathrm{~S}_{2}$ (877.02): C: 57.52\%, H: 6.44\%, N: 3.19\%, S: 7.31\%; Found: C: 57.85\%, H: 7.76\%, N: 3.18\%, S: 7.03\%; TLC R 0.67 (EtOAc/hexane, 1/4). 
2,2-Dimethyl-propionic acid 2-[2-(2,6-bis-allyloxy-4-\{2-[2-(3,5-bis-allyloxy-4-\{2-[2-(2, 2-dimethyl-propionyloxy)-ethoxy]-ethoxy\}-benzoylamino)-ethyldisulfanyl]-ethylcarbamoyl \}-phenoxy)-ethoxy]-ethyl ester (7b)

In a dry $50 \mathrm{~mL}$, two-necked, round-bottomed flask was placed molybdenum dioxydichloride ( $2 \mathrm{mg}, 0.01 \mathrm{mmol}, 8.7 \mathrm{~mol} \%, 0.045$ equiv) in $15 \mathrm{~mL}$ of anhydrous dichloromethane. To the above solution, pivalic anhydride (112 $\mu \mathrm{L}, 0.55 \mathrm{mmol}, 2.4$ equiv) was slowly added at ambient temperature. After for $10 \mathrm{~min}$, a solution of corresponding alcohol $\left(90 \mathrm{mg}, 0.115 \mathrm{mmol}\right.$ in $\mathrm{CH}_{2} \mathrm{Cl}_{2}$, $2 \mathrm{~mL}$ ) was slowly added to the above dark bluish green solution and the reaction mixture were stirred for 48 hours at room temperature. After completion, reaction mixture was quenched with cold, saturated aqueous $\mathrm{NH}_{4} \mathrm{Cl}$ solution $(5 \mathrm{~mL})$ and extracted with dichloromethane $(2 \times 5 \mathrm{~mL})$. The separated organic layer was washed with brine, dried $\left(\mathrm{MgSO}_{4}\right)$, filtered, and evaporated. The crude product was purified by column chromatography. The product obtained as colorless oil (92 mg, $84 \%$ ) was characterized by routine spectroscopic methods.

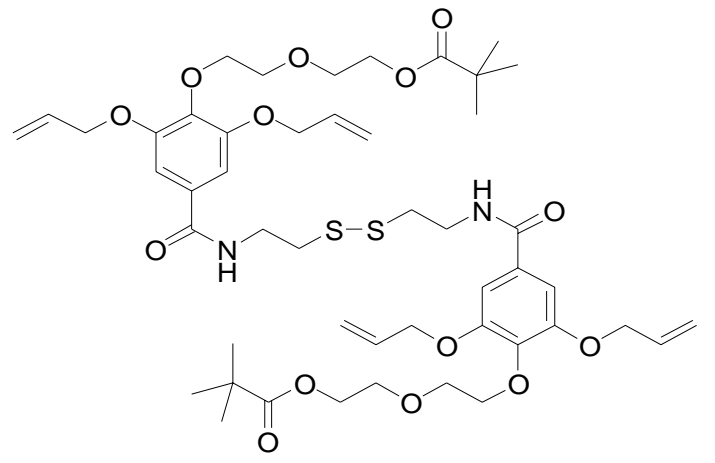

Data: ${ }^{1} \mathrm{H}$ NMR (500 MHz, $\left.\mathrm{CDCl}_{3}\right) 7.22(\mathrm{t}, J=6.6,1 \mathrm{H})$, 7.08 (s, 2H), 6.00-5.92 (m, 2H), 5.35 (dd, $J=17.0,1.0$, 2H), 5.21 (dd, $J=10.5,1.0,2 \mathrm{H}), 4.51$ (d, $J=5.1,4 \mathrm{H})$, 4.19-4.15 (m, 4H), $3.78(\mathrm{t}, J=5.1,2 \mathrm{H}), 3.76-3.72(\mathrm{~m}$, $4 \mathrm{H}), 2.95(\mathrm{t}, J=6.2,2 \mathrm{H}), 1.17(\mathrm{~s}, 9 \mathrm{H}) ;{ }^{13} \mathrm{C}$ NMR $(125$ $\left.\mathrm{MHz}, \mathrm{CDCl}_{3}\right)$ 178.48, 167.37, 152.33, 141.17, 132.89, 129.24, 117.62, 106.69, 72.36, 70.45, 69.99, 69.08, 63.57, 39.36, 38.66, 37.90, 27.12; IR (CCl $) 3303$ (w), 2931 (m), 1730 (m), 1650 (m), 1581 (m), 1492 (m), 1420 (m), 1330 (m), 1284 (m), 1225 (w), 1132 (s), 930 (w); MS (70 eV) 482 ((M/2)+1 , 98), 466 (50), 448 (40), 404 (35), 294 (35), 232 (40), 215 (25), 129 (100); Anal. Calcd. For $\mathrm{C}_{48} \mathrm{H}_{68} \mathrm{~N}_{2} \mathrm{O}_{14} \mathrm{~S}_{2}$ (961.18): C: 59.98\%, H: 7.13\%, N: 2.91\%, S: 6.67\%; Found: C: 59.57\%, H: 7.69\%, N: 2.54\%, S: 6.09\%; TLC R 0.85 (EtOAc/hexane, 1/4).

Carbonic acid 2(2-\{2,6-bis-llyloxy-4-[2-(2-\{3,5-bis-allyloxy-4-[2-(2-tert-butoxycarb onyloxy-ethoxy)-ethoxy]-benzoylamino\}-ethyldisulfanyl)-ethylcarbamoyl]-phenoxy\}-ethoxy )-ethyl ester tert-butyl ester (7c)

In a dry $50 \mathrm{~mL}$, two-necked, round-bottomed flask was placed molybdenum dioxydichloride 
( $2 \mathrm{mg}, 0.01 \mathrm{mmol}, 8.5 \mathrm{~mol} \%, 0.044$ equiv ) in $15 \mathrm{~mL}$ of anhydrous dichlorometane. To the above solution, di-t-butyl-dicarbonate $(134 \mu \mathrm{L}, 0.58 \mathrm{mmol}, 2.5$ equiv) was slowly added at ambient temperature. After for $10 \mathrm{~min}$, a solution of corresponding alcohol $\left(93 \mathrm{mg}, 0.118 \mathrm{mmol}\right.$ in $\mathrm{CH}_{2} \mathrm{Cl}_{2}$, $2 \mathrm{~mL}$ ) was slowly added to the above dark bluish green solution and the reaction mixture were refluxed for 48 hours. After completion, reaction mixture was quenched with cold, saturated aqueous $\mathrm{NH}_{4} \mathrm{Cl}$ solution $(5 \mathrm{~mL})$ and extracted with dichloromethane $(2 \times 5 \mathrm{~mL})$. The separated organic layer was washed with brine, dried $\left(\mathrm{MgSO}_{4}\right)$, filtered, and evaporated. The crude product was purified by column chromatography. The product obtained as colorless oil (104 mg, $90 \%$ ) was characterized by routine spectroscopic methods.

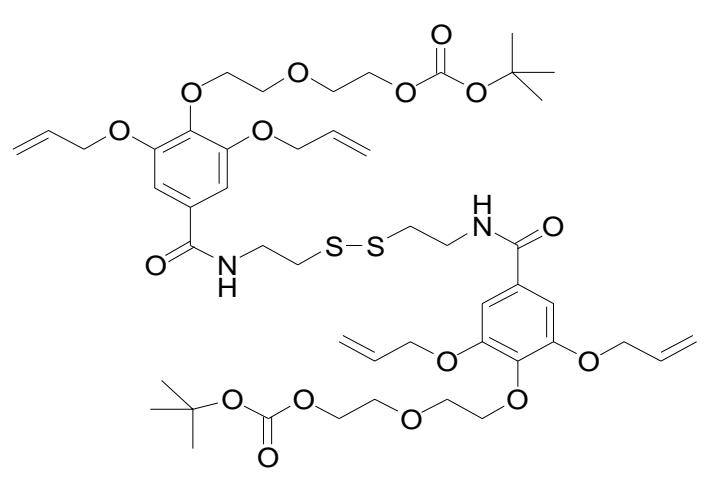

${ }^{1} \mathrm{H}$ NMR (500 MHz, $\left.\mathrm{CDCl}_{3}\right) 7.09(\mathrm{t}, J=6.2,1 \mathrm{H}), 7.07$ (s, 2H), 6.08-5.94 (m, 2H), $5.36(\mathrm{dd}, J=17.2,1.0,2 \mathrm{H})$, $5.23(\mathrm{dd}, J=10.8,1.0,2 \mathrm{H}), 4.53(\mathrm{~d}, J=5.1,4 \mathrm{H}), 4.18$, $(\mathrm{t}, J=5.0,4 \mathrm{H}), 3.79(\mathrm{t}, J=5.0,2 \mathrm{H}), 3.77-3.75(\mathrm{~m}, 4 \mathrm{H})$, $2.96(\mathrm{t}, J=6.3,2 \mathrm{H}), 1.46(\mathrm{~s}, 3 \mathrm{H}) ;{ }^{13} \mathrm{C} \mathrm{NMR}(125 \mathrm{MHz}$, $\left.\mathrm{CDCl}_{3}\right)$ 167.36, 153.49, 152.38, 141.19, 132.93, 129.28, 117.69, 106.67, 82.08, 72.38, 70.45, 70.04, 68.92, 66.07, 39.34, 37.92, 27.73; IR ( $\left.\mathrm{CCl}_{4}\right) 3354$ (w), 2929 (w), 1742 (s), 1650 (m), 1581 (m), 1492 (s), 1420 (m), 1368 (m), 1330 (s), 1279 (s), 1133 (s), 929 (w); MS (70eV) 498 (M/2 , <1), 453 (15), 397 (100), 382 (70), 320 (45), 234 (40), 215 (35), 199 (20); Anal. Calcd. For $\mathrm{C}_{48} \mathrm{H}_{68} \mathrm{~N}_{2} \mathrm{O}_{16} \mathrm{~S}_{2}$ (993.18): C: 58.05\%, H: 6.90\%, N: 2.82\%, S: 6.46\%; Found: C: 57.79\%, H: 7.19\%, N: 2.73\%, S: 6.12\%; TLC R 0.72 (EtOAc/hexane, 1/4).

\section{3,5-Bis-[3-(2,2-dimethyl-propionyloxy)-2-hydroxy-propoxy]-4-\{2-[2-(tetrahydro-pyran-2-yl oxy)-ethoxy]-ethoxy\}-benzoic acid methyl ester (8a)}

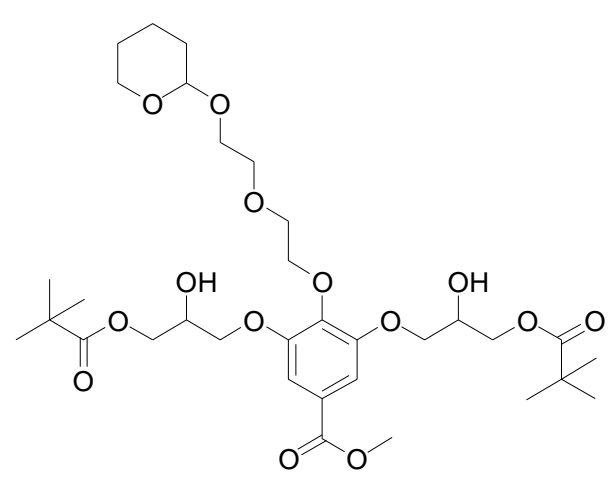

In a dry $50 \mathrm{~mL}$, two-necked, round bottom flask was placed $\mathrm{MoO}_{2} \mathrm{Cl}_{2}$ (14 mg, $0.07 \mathrm{mmol}, 15 \mathrm{~mol} \%$ ) in $15 \mathrm{~mL}$ of anhydrous $\mathrm{CH}_{2} \mathrm{Cl}_{2}$. To the above solution, pivalic anhydride (90 mg, $0.48 \mathrm{mmol}$ ), was slowly added at ambient temperature. After for $45 \mathrm{~min}$, a solution of the corresponding tetraol (123 mg, $0.24 \mathrm{mmol}$ in $\mathrm{CH}_{2} \mathrm{Cl}_{2}, 5 \mathrm{~mL}$ ) was slowly added to the above dark bluish green solution 
and the reaction mixture were stirred at ambient temperature for 32 hours. After completion, reaction mixture was quenched with cold, saturated aqueous $\mathrm{NaHCO}_{3}$ solution $(5 \mathrm{~mL})$ and extracted with dichloromethane $(2 \times 15 \mathrm{~mL})$. The separated organic layer was washed with brine, dried $\left(\mathrm{MgSO}_{4}\right)$, filtered and evaporated. The crude product was purified by column chromatography on silica gel. The product-8a was obtained as colorless oil (118 mg, $73 \%)$ : ${ }^{1} \mathrm{HNMR}\left(500 \mathrm{MHz}, \mathrm{d}_{6}\right.$-DMSO) 7.24 (s, 2H), 5.31(bs, 2H), 4.53-4.52 (t, $\left.J=3.5 \mathrm{~Hz}, 1 \mathrm{H}\right), 4.14-4.08$ $(\mathrm{m}, 6 \mathrm{H}), 4.05-3.99(\mathrm{~m}, 6 \mathrm{H}), 3.82(\mathrm{~s}, 3 \mathrm{H}), 3.73(\mathrm{~m}, 4 \mathrm{H}), 3.58-3.56(\mathrm{t}, J=4.4 \mathrm{~Hz}, 2 \mathrm{H}), 3.46-3.34(\mathrm{~m}$,

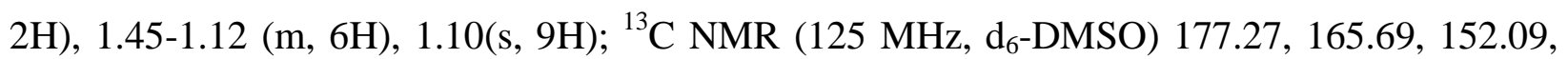
141.94, 124.49, 108.20, 98.03, 71.98, 70.24, 69.85, 69.65, 66.75, 66.06, 64.93, 61.17, 52.18, 30.15, 26.82, 24.98, 19.05; IR $\left(\mathrm{CH}_{2} \mathrm{Cl}_{2}\right) 3466$ (br, m), 2926 (m), 1722 (s), 1648 (m), 1590 (m), 1502 (m), 1481 (m), 1365 (m), 1342 (m), 1124 (m), 1035 (m), 938 (m), 886 (m); MS (70 eV) 672 $\left(\mathrm{M}^{+}, 5\right), 533$ (7), 455 (7), 243 (20), 159 (100); HRMS Calcd for $\left(\mathrm{C}_{33} \mathrm{H}_{52} \mathrm{O}_{14}\right) 672.3357$ found 672.3352; TLC R 0.49 (EtOAc/hexane, 1/2)

\section{3,5-Bis-(3-benoyloxy)-2-hydroxy-propoxy)-4-\{2-[2-(tetrahydro-pyran-2-yloxy)-ethoxy]-etho xy -benzoic acid methyl ester $(8 b)$}

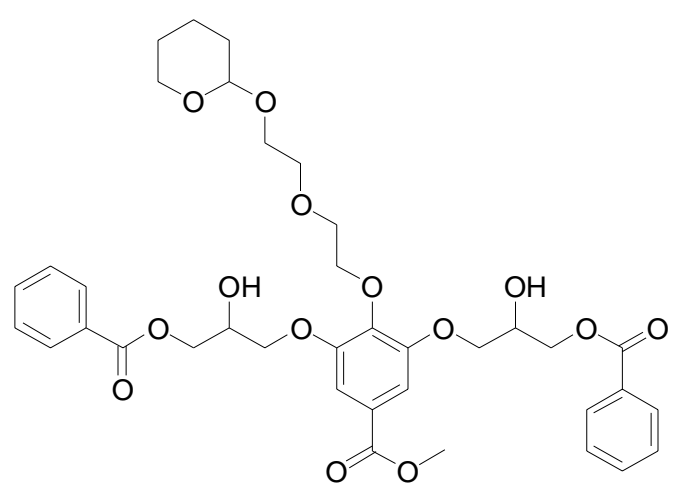

In a dry $50 \mathrm{~mL}$, two-necked, round bottom flask was placed $\mathrm{MoO}_{2} \mathrm{Cl}_{2}(23 \mathrm{mg}, 0.12 \mathrm{mmol}, 15 \mathrm{~mol} \%)$ in 15 $\mathrm{mL}$ of anhydrous $\mathrm{CH}_{2} \mathrm{Cl}_{2}$. To the above solution, benzoic anhydride (179 $\mathrm{mg}, 0.79 \mathrm{mmol})$, was slowly added at ambient temperature. After for $45 \mathrm{~min}$, a solution of the corresponding tetraol $(200 \mathrm{mg}, 0.39$ mmol in $\mathrm{CH}_{2} \mathrm{Cl}_{2}, 5 \mathrm{~mL}$ ) was slowly added to the above dark bluish green solution and the reaction mixture were stirred at ambient temperature for 47 hours. The reaction mixture was quenched with cold, saturated aqueous $\mathrm{NaHCO}_{3}$ solution $(5 \mathrm{~mL})$ and extracted with dichloromethane $(2 \times 15 \mathrm{~mL})$. The separated organic layer was washed with brine, dried $\left(\mathrm{MgSO}_{4}\right)$, filtered and evaporated. The crude product was purified by column chromatography on silica gel. The product-8b was obtained as colorless oil $(237 \mathrm{mg}, 85 \%):{ }^{1} \mathrm{H}$ NMR (500 MHz, d 6 -DMSO) 8.01-7.99 (dd, $J=7.1 \mathrm{~Hz}, J=1.3 \mathrm{~Hz}, 4 \mathrm{H}), 7.66-7.63(\mathrm{~m}, 2 \mathrm{H})$, 7.53-7.50 (m, 4H), 7.29 (s, 2H), 5.48 (bs, 2H), 4.46-4.34 (m, 5H), 4.21-4.19 (m, 2H), 4.15-4.10 (m, 6H), $3.80(\mathrm{~s}, 3 \mathrm{H}), 3.70-3.61(\mathrm{~m}, 4 \mathrm{H}), 3.56-3.54(\mathrm{t}, J=4.3 \mathrm{~Hz}), 3.42-3.30(\mathrm{~m}, 2 \mathrm{H}), 1.59-1.34(\mathrm{~m}$, 
$6 \mathrm{H}) ;{ }^{13} \mathrm{C}$ NMR (125 MHz, d $\mathrm{d}_{6}$-DMSO) 165.70, 165.66, 152.08, 141.97, 133.26, 129.67, 129.24, $128.78,124.52,108.35,98.03,72.03,70.34,69.88,69.64,66.89,66.03,65.89,61.16,52.12$, 30.11, 24.94, 19.03; IR $\left(\mathrm{CH}_{2} \mathrm{Cl}_{2}\right) 3472$ (br, m), 2877 (m), 1723 (s), 1650 (m), 1589 (m), $1503(\mathrm{~m})$, 1342 (m), 1211 (m), 1114 (m), 1033 (m), 937(m), 869 (m); MS (70 eV) $712\left(\mathrm{M}^{+}, 5\right), 283$ (20), 179 (100), 105 (50), 85(22); TLC R 0.39 (EtOAc/hexane, 1/2).

\section{References:}

(1) (a) Nie, J.; Zhao, Z.; Xu, J.; Liu, D. J. Chem. Res. Synop. 1999, 2, 160-161. (b) Nakamura, K.; Kawasaki, M.; Ohno, A. Bull. Chem. Soc. Jpn. 1994, 67, 3053-3056.

(2) Modena, G.; Rivetti, F.; Scorrano, G.; Tonellato, U. J. Am. Chem. Soc. 1977, 99, 3392-3395.

(3) Saigo, K. Bull. Chem. Soc. Jpn. 1977, 50, 1863-1866.

(4) Aida, T. Chem. Lett. 1975; 29-32.

(5) Hansen, G. R.; Burg, T. E. J. Heterocycl. Chem. 1967, 4, 653-656.

(6) (a) Michel, D.; Schlosser, M. Tetrahedron 2000, 56, 4253-4260. (b) Isobe, T.; Ishikawa, T. J. Org. Chem. 1999, 64, 6984-6988.

(7) Houlihan, F.; Bouchard, F.; Frechet, J. M. J.; Willson, C. G. Can. J. Chem. 1985, 63, 153-163.

(8) Fujisawa, T.; Mori, T.; Fukumoto, K.; Sato, T. Chem. Lett. 1982, 1891-1894.

(9) Kita, Y.; Maeda, H.; Takahashi, F.; Fukui, S. J Chem. Soc. Perkin Trans. 1993, 21, 2639-2650.

(10) Trahanovsky, W. S.; Ong, C. C. J. Am. Chem. Soc. 1970, 92, 7174-7177.

(11) Kim, S.; Yang, S. Synth. Commun. 1981, 11, 121-124.

(12) Bandgar, B. P.; Kamble, V. T.; Sadavarte, V. S.; Uppalla, L. S. Syn. Lett. 2002, 5; 735-738.

(13) Falck, J. R.; Bhatt, R. K.; Ye, J. J. Amer. Chem. Soc. 1995, 117, 5973-5982.

(14) Iida M. Agric. Biol. Chem. 1977, 41, 2471.

(15) Kaminska, J. E.; Kaminski, Z. J.; Gora, J. Synthesis 1999, 4, 593-596.

(16) Orita, A.; Tanahashi, C.; Kakuda, A.; Otera, J. J. Org. Chem. 2001, 66, 8926.

(17) Rosado-Lojo, O.; Hancock, C. K.; Danti, A. J. Org. Chem. 1966, 31, 1899.

(18) Strazzolini, P.; Arche, M. G. D.; Giumanini, A. G.; Tetrahedron Lett. 1998, 50, 9255.

(19) Hans, J. J.; Driver, R. W.; Burke, S. D.; J. Org. Chem. 2000, 65, 2114.

(20) Alcock; T. J. Chem. Soc. A 1968, 1873. 
(21) Berlin, K. D.; Gower, L. H.; White, J. W.; Gibbs, D. E.; Sturm, G. P. J. Org. Chem. 1962, 27, 3595.

(22) Kuo, M.-Y.; Liu, K.-T.; J. Org. Chem. 1987, 52, 2927.

(23) Bellucci, G.; Bianchini, R.; Chiappe, C.; Marioni, F.; Catalano, D. Tetrahedron 1988, 44, 4863-4870.

(24) (a) Ram, B.; Roy, J.; Sarkar, A. Indian J. Chem. Sect. B 1988, 27, 665. (b) Jin, T.-S.; Ma, Y.-R.; Li, T.-S.; Zhang, Z.-H.; Duan, G.-B. Indian J. Chem. Sect. B 1999, 38, 109-110.

(25) Kim, S.; Yang, S. Synth. Commun. 1981, 11, 121-124.

(26) Singh, A. K.; Sonar, S. M. Synth. Commun. 1985, 15, 1113-1122.

(27) (a) DeRuiter, J.; Swearingen, B. E.; Wandrekar, V.; Mayfield, C. A. J. Med. Chem. 1989, 32, 1033-1038. (b) Takai, H. Chem. Pharm. Bull. 1978, 26, 1966-1972.

(28) Patel, M.; Ko, S. S.; McHugh, R. J.; Markwalder, J. A.; Srivastava, A. S.; Cordova, B. C.; Klabe, R. M.; Erickson-Viitanen, S.; Trainor, G. L.; Seitz, S. P. Bioorg. Med. Chem. Lett. 1999, 9, 2805-2810.

(29) DeRuiter, J.; Swearingen, B. E.; Wandrekar, V.; Mayfield, C. A. J. Med. Chem. 1989, 32, 1033-1038.

(30) Baudoin, O.; Claveau, F.; Thoret, S.; Herrbach, A.; Guenard, D.; Gueritte. Bioorg. Med. Chem. 2002, 11, 3395 - 3400.

(31) Kamijo, T.; Yamamoto, R.; Harada, H.; lizuka, K. Chem. Pharm. Bull. 1982, 30, 4242-4244.

(32) Penn, J. H.; Liu, F. J. Org. Chem. 1994, 59, 2608-2612.

(33) Kobayashi, K.; Koyama, E.; Kono, C.; Namatane, K.; Nakamura, K.; Furukawa, N. J. Org. Chem. 2001, 66, 2085 - 2090.

(34) Kondo, K.; Iida, T.; Fujita, H.; Suzuki, T.; Yamaguchi, K.; Murakami. Tetrahedron 2000, 56, $8883-8892$.

(35) Shangguan, N.; Katukojvala, S.; Greenberg, R.; Williams, L. J. J. Am. Chem. Soc. 2003, 125, 7754-7755.

(36) Wu, S.; Lee, S.; Beak, P. J. Am. Chem. Soc. 1996, 118, 715-721.

(37) Liden, A.; Roussel, C.; Liljefor, T.; Chanon, M.; Carter, R. E.; Metzger, J.; Sandstrom. J. J. Am. Chem. Soc. 1976, 98, 2853-2860.

(38) Newcomb, M.; Varick, T. R.; Goh, S.-H. J. Am. Chem. Soc. 1990, 112, 5186-5193. 
(39) Nomura, R.; Nakano, T.; Yamada, Y.; Matsuda, H. J. Org. Chem. 1991, 56, 4076-4078.

(40) Nakagawa, H.; Nagano, T.; Higuchi, T. Org. Lett. 2001, 3, 1805-1807.

(41) Pirkle, W.H.; Simmons, K. A.; Boeder, C. W. J. Org. Chem. 1979, 26, 4891-4896.

(42) Stavropoulos, P.; Muetterties, M. C.; Carrie, M.; Holm, R. H. J. Am. Chem. Soc. 1991, 113, 8485-8492.

(43) Imamoto, T.; Kodera, M.; Yokoyama, M. Synthesis 1982, 2, 134-136.

(44) Inoue, T.; Takeda, T.; Kambe, N.; Ogawa, A.; Ryu, I.; Sonoda, N. J. Org. Chem. 1994, 59, 5824-5827.

(45) (a) Gennari, C.; Cozzi, P. G. Tetrahedron 1988, 44, 5965-5974. (b) Gurudutt, K. N.; Rao, S.; Srinivas, P.; Srinivas, S. Tetrahedron 1995, 51, 3045-3050.

(46) (a) Dean, C. S.; Tarbell, D. S. J. Org. Chem. 1971, 36, 1180-1183. (b) Stanley, R. L.; Tarbell, D. S. J. Org. Chem. 1977, 42, 3686-3690.

(47) Piepers, O.; Kellogg, R. M. J. Chem. Soc. Chem. Commun. 1980, 23, 1147-1149.

(48) McCasland, G. E.; Horswill, E. C. J. Am. Chem. Soc. 1954, 76, 1654-1656.

(49) Orita, A.; Sakamoto, K.; Hamada, Y.; Mitsutome, A.; Otera, J. Tetrahedron 1999, 55, 28992910.

(50) Orita, A.; Sakamoto, K.; Hamada, Y.; Mitsutome, A.; Otera, J. Tetrahedron 1999, 55, 28992910.

(51) Reynolds, K. A.; O’Hagan, D.; Gani, D.; Robinson, J. A. J. Chem. Soc. Perkin Trans. 1 1988, 3195-3208.

(52) Orita, A.; Tanahashi, C.; Kakuda, A.; Otera, J. Angew. Chem. Int. Ed. Engl. 2000, 39, 28772879; Angew. Chem. 2000, 112, 2999-3001.

(53) (a) Budzikiewicz, H.; Dallakian, P.; Griesbeck, A. G.; Heckroth, H. J. Mass. Spectrom. 1998, 33, 1256-1260. (b) Barker, J.; Cook, S. L.; Lasterra-Sanche M. E.; Thomas, S. E. J. Chem. Soc. Chem. Commun. 1992, 11, 830-832.

(54) Bretschneider, T.; Miltz, W.; Münster, P.; Steglich, W. Tetrahedron 1988, 44, 5403-5414.

(55) Braghiroli, D.; Bella, M. D. Tetrahedron: Asymmetry 1996, 7, 2145-2150.

(56) (a) House, H. O. J. Am. Chem. Soc. 1973, 95, 3310-3324. (b) Yanagisawa, M.; Shimamura, T.; Iida, D.; Matsuo, J.-I; Mukaiyama, T. Chem. Pharm. Bull. 2000, 48, 1838-1840. (c) Basselier,J.-J. Bull. Soc. Chim. Fr. 1965; 2988-2994. 
(57) (a) Saladino, R.; Crestini, C.; Occhionero, F.; Nicoletti, R. Tetrahedron 1995, 51, 3607-3616. (b) Lazrek, H. B.; Engels, J. W.; Pfleiderer, W. Nucleosides Nucleotides 1998, 17, 1851-1856.

(58) Tsui, H.-C.; Paquett, L. A. J. Org. Chem. 1998, 26, 9968-9977.

(59) Liptak, A.; Nanasi, P.; Neszmelyi, A.; Wagner, H. Carbohydr. Res. 1980, 86, 133-136.

(60) McPhail, D. R.; Lee, J. R.; Fraser-Reid, B. J. Am. Chem. Soc. 1992, 114, 1905-1906.

(61) Khan, R.; Konowicz, P. A.; Gardossi, L.; Matulova, M.; de Gennarao, S. Aust. J. Chem. 1996, 49, 293-298.

(62) (a) Hirayama, F.; Yamanaka, M.; Horikawa, T.; Uekama, K. Chem. Pharm. Bull. 1995, 43, 130-136.

(63) Sommer, A. J.; Katon, J. E. Appl.Spectrosc. 1991, 45; 1633-1640.

(64) Ashram, M.; Miller, D. O.; Georghiou, P. E. J. Chem. Soc. Perkin Trans.1 2002, 12, 1470-1476.

(65) Takahashi, H.; Tsubuki, T.; Higashiyama, K. Chem. Pharm. Bull. 1991, 39, 3136-3139.

(66) Aitken, R. A.; Armstrong, D. P.; Galt, R. H. B.; Mesher, S. T. E. J. Chem. Soc. Perkin Trans. 1 1997, 6, 935-944.

(67) Lewanowicz, A.; Lipinski, J.; Siedlecka, R.; Skarzewski, J.; Baert, F. Tetrahedron 1998, 54, 6571-6586.

(68) Braghiroli, D.; Bella, M. D. Tetrahedron: Asymmetry 1996, 7, 2145-2150.

(69) Ranu, B. C.; Dutta, P.; Sarkar, A. J. Chem. Soc. Perkin Trans. 1 2000, 14, 2223-2226.

(70) Morcuende, A.; Ors, M.; Valverde, S.; Herradón, B. J. Org. Chem. 1996, 61, 5264-5270.

(71) Benson, O.; Demirdji, S. H.; Haltiwanger, R. C.; Koch, T. H. J. Am. Chem. Soc. 1991, 113, 8879-8886.

(72) Saigo, K.; Usui, M.; Kinuchi, K.; Shimada, E.; Mukaiyama, T. Bull. Chem. Soc. Jpn. 1977, 50,4683 .

(73) Murata, S.; Noyori, R.; Tetrahedron Lett. 1981, 22, 2107. 